\title{
Which simple types have a unique inhabitant?
}

\author{
Gabriel Scherer Didier Rémy \\ INRIA \\ \{gabriel.scherer,didier.remy\}@inria.fr
}

\begin{abstract}
We study the question of whether a given type has a unique inhabitant modulo program equivalence. In the setting of simplytyped lambda-calculus with sums, equipped with the strong $\beta \eta$ equivalence, we show that uniqueness is decidable. We present a saturating focused logic that introduces irreducible cuts on positive types "as soon as possible". Backward search in this logic gives an effective algorithm that returns either zero, one or two distinct inhabitants for any given type. Preliminary application studies show that such a feature can be useful in strongly-typed programs, inferring the code of highly-polymorphic library functions, or "glue code" inside more complex terms.
\end{abstract}

Categories and Subject Descriptors F.3.3 [Logics and Meanings of Programs]: Studies of Program Constructs-Type structure

General Terms Languages, Theory

Keywords Unique inhabitants, proof search, simply-typed lambdacalculus, focusing, canonicity, sums, saturation, code inference

\section{Introduction}

In this article, we answer an instance of the following question: "Which types have a unique inhabitant"? In other words, for which type is there exactly one program of this type? Which logical statements have exactly one proof term?

To formally consider this question, we need to choose one specific type system, and one specific notion of equality of programs - which determines uniqueness. In this article, we work with the simply-typed $\lambda$-calculus with atoms, functions, products and sums as our type system, and we consider programs modulo $\beta \eta$ equivalence. We show that unique inhabitation is decidable in this setting; we provide and prove correct an algorithm to answer it, and suggest several applications for it. This is only a first step: simplytyped calculus with sums is, in some sense, the simplest system in which the question is delicate enough to be interesting. We hope that our approach can be extended to richer type systems - with polymorphism, dependent types, and substructural logics.

The present version is identical to the short version, except for the Appendix which contains a detailed presentation of the algorithm (Appendix A), and proof arguments for the formal results (Append $B)$.

\subsection{Why unique?}

We see three different sources of justification for studying uniqueness of inhabitation: practical use of code inference, programming language design, and understanding of type theory.

In practice, if the context of a not-yet-written code fragment determines a type that is uniquely inhabited, then the programming system can automatically fill the code. This is a strongly principal form of code inference: it cannot guess wrong. Some forms of code completion and synthesis have been proposed (Perelman, Gulwani, Ball, and Grossman 2012; Gvero, Kuncak, Kuraj, and Piskac
2013), to be suggested interactively and approved by the programmer. Here, the strong restriction of uniqueness would make it suitable for a code elaboration pass at compile-time: it is of different nature. Of course, a strong restriction also means that it will be applicable less often. Yet we think it becomes a useful tool when combined with strongly typed, strongly specified programming disciplines and language designs - we have found in preliminary work (Scherer 2013) potential use cases in dependently typed programming. The simply-typed lambda-calculus is very restricted compared to dependent types, or even the type systems of ML, System $\mathrm{F}$, etc. used in practice in functional programming languages; but we have already found a few examples of applications (Section 6). This shows promises for future work on more expressive type systems.

For programming language design, we hope that a better understanding of the question of unicity will let us better understand, compare and extend other code inference mechanisms, keeping the question of coherence, or non-ambiguity, central to the system. Type classes or implicits have traditionally been presented (Wadler and Blott 1989; Stuckey and Sulzmann 2002; Oliveira, Schrijvers, Choi, Lee, Yi, and Wadler 2014) as a mechanism for elaboration, solving a constraint or proof search problem, with coherence or non-ambiguity results proved as a second step as a property of the proposed elaboration procedure. Reformulating coherence as a unique inhabitation property, it is not anymore an operational property of the specific search/elaboration procedure used, but a semantic property of the typing environment and instance type in which search is performed. Non-ambiguity is achieved not by fixing the search strategy, but by building the right typing environment from declared instances and potential conflict resolution policies, with a general, mechanism-agnostic procedure validating that the resulting type judgments are uniquely inhabited.

In terms of type theory, unique inhabitation is an occasion to take inspiration from the vast literature on proof inhabitation and proof search, keeping relevance in mind: all proofs of the same statement may be equally valid, but programs at a given type are distinct in important and interesting ways. We use focusing (Andreoli 1992), a proof search discipline that is more canonical (enumerates less duplicates of each proof term) than simply goal-directed proof search, and its recent extension into (maximal) multi-focusing (Chaudhuri, Miller, and Saurin 2008).

\subsection{Example use cases}

Most types that occur in a program are, of course, not uniquely inhabited. Writing a term at a type that happens to be uniquely inhabited is a rather dull part of the programming activity, as they are no meaningful choices. While we do not hope unique inhabitants would cure all instances of boring programming assignment, we have identified two areas where they may be of practical use:

- inferring the code of highly parametric (strongly specified) auxiliary functions 
- inferring fragments of glue code in the middle of a more complex (and not uniquely determined) term

For example, if you write down the signature of flip $\forall \alpha \beta \gamma .(\alpha \rightarrow \beta \rightarrow \gamma) \rightarrow(\beta \rightarrow \alpha \rightarrow \gamma)$ to document your standard library, you should not have to write the code itself. The types involved can be presented equivalently as simple types, replacing prenex polymorphic variables by uninterpreted atomic types $(\mathrm{X}, \mathrm{Y}$, Z...). Our algorithm confirms that $(X \rightarrow Y \rightarrow Z) \rightarrow(Y \rightarrow$ $X \rightarrow Z)$ is uniquely inhabited and returns the expected program same for curry and uncurry, const, etc.

In the middle of a term, you may have forgotten whether the function proceedings excepts a conf as first argument and a year as second argument, or the other way around. Suppose a language construct ?! that infers a unique inhabitant at its expected type (and fails if there are several choices), understanding abstract types (such as year) as uninterpreted atoms. You can then write (?! proceedings icfp this_year), and let the programming system infer the unique inhabitant of either (conf $\rightarrow$ year $\rightarrow$ proceedings $) \rightarrow$ (conf $\rightarrow$ year $\rightarrow$ proceedings $)$ or (conf $\rightarrow$ year $\rightarrow$ proceedings $) \rightarrow$ (year $\rightarrow$ conf $\rightarrow$ proceedings) depending on the actual argument order - it would also work for conf $*$ year $\rightarrow$ proceedings, etc.

\subsection{Aside: Parametricity?}

Can we deduce unique inhabitation from the free theorem of a sufficiently parametric type? We worked out some typical examples, and our conclusion is that this is not the right approach. Although it was possible to derive uniqueness from a type's parametric interpretation, proving this implication (from the free theorem to uniqueness) requires arbitrary reasoning steps, that is, a form of proof search. If we have to implement proof search mechanically, we may as well work with convenient syntactic objects, namely typing judgments and their derivations.

For example, the unary free theorem for the type of composition $\forall \alpha \beta \gamma .(\alpha \rightarrow \beta) \rightarrow(\beta \rightarrow \gamma) \rightarrow(\alpha \rightarrow \gamma)$ tells us that for any sets of terms $S_{\alpha}, S_{\beta}, S_{\gamma}$, if $f$ and $g$ are such that, for any $a \in S_{\alpha}$ we have $f a \in S_{\beta}$, and for any $b \in S_{\beta}$ we have $g b \in S_{\gamma}$, and if $t$ is of the type of composition, then for any $a \in S_{\alpha}$ we have $t f g a \in S_{\gamma}$. The reasoning to prove unicity is as follows. Suppose we are given functions (terms) $f$ and $g$. For any term $a$, first define $S_{\alpha} \stackrel{\text { def }}{=}\{a\}$. Because we wish $f$ to map elements of $S_{\alpha}$ to $S_{\beta}$, define $S_{\beta} \stackrel{\text { def }}{=}\{f a\}$. Then, because we wish $g$ to map elements of $S_{\beta}$ to $S_{\gamma}$, define $S_{\gamma} \stackrel{\text { def }}{=}\{g(f a)\}$. We have that $t f g a$ is in $S_{\gamma}$, thus $t f g$ is uniquely determined as $\lambda a . g(f a)$.

This reasoning exactly corresponds to a (forward) proof search for the type $\alpha \rightarrow \gamma$ in the environment $\alpha, \beta, \gamma, f: \alpha \rightarrow \beta, g$ : $\beta \rightarrow \gamma$. We know that we can always start with a $\lambda$-abstraction (formally, arrow-introduction is an invertible rule), so introduce $x$ : $\alpha$ in the context and look for a term of type $\gamma$. This type has no head constructor, so no introduction rules are available; we shall look for an elimination (function application or pair projection). The only elimination we can perform from our context is the application $f x$, which gives a $\beta$. From this, the only elimination we can perform is the application $g(f x)$, which gives a $\gamma$. This has the expected goal type: our full term is $\lambda x . g(f x)$. It is uniquely determined, as we never had a choice during term construction.

\subsection{Formal definition of equivalence}

We recall the syntax of the simply-typed lambda-calculus types (Figure 1), terms (Figure 2) and neutral terms. The standard typing judgment $\Delta \vdash t: A$ is recalled in Figure 3 , where $\Delta$ is a general context mapping term variables to types. The equivalence relation we consider, namely $\beta \eta$-equivalence, is defined as the least congruence satisfying the equations of Figure 4. Writing $t: A$ in an equivalence rule means that the rule only applies when the subterm $t$ has type $A$-we only accept equivalences that preserve well-typedness.

\begin{tabular}{|c|c|c|}
\hline \multicolumn{2}{|c|}{$A, B, C, D::=$} & types \\
\hline & $X, Y, Z$ & atoms \\
\hline & $P, Q$ & positive types \\
\hline & $N, M$ & negative types \\
\hline & $::=A+B$ & strict positive \\
\hline V,$M$ & $::=A \rightarrow B \mid A * B$ & strict negative \\
\hline,$Q_{\mathrm{at}}$ & $::=P, Q \mid X, Y, Z$ & positive or atom \\
\hline$M$ & $::=N, M \mid X, Y, Z$ & negative or atom \\
\hline
\end{tabular}

Figure 1. Types of the simply-typed calculus

\begin{tabular}{|c|c|c|}
\hline \multirow{8}{*}{$t, u, r::=$} & & terms \\
\hline & $x, y, z$ & variables \\
\hline & $\lambda x . t$ & $\lambda$-abstraction \\
\hline & $t u$ & application \\
\hline & $(t, u)$ & pair \\
\hline & $\pi_{i} t$ & projection $(i \in\{1,2\})$ \\
\hline & $\sigma_{i} t$ & sum injection $(i \in\{1,2\})$ \\
\hline & $\delta\left(t, x_{1} . u_{1}, x_{2} . u_{2}\right)$ & sum elimination (case split) \\
\hline$m:=$ & $y, z\left|\pi_{i} n\right| n t$ & neutral terms \\
\hline
\end{tabular}

Figure 2. Terms of the lambda-calculus with sums

$$
\begin{array}{cc}
\frac{\Delta, x: A \vdash t: B}{\Delta \vdash \lambda x \cdot t: A \rightarrow B} & \frac{\Delta \vdash t: A \rightarrow B \quad \Delta \vdash u: A}{\Delta \vdash t u: B} \\
\frac{\Delta \vdash t: A \quad \Delta \vdash u: B}{\Delta \vdash(t, u): A * B} & \frac{\Delta \vdash t: A_{1} * A_{2}}{\Delta \vdash \pi_{i} t: A_{i}} \\
\Delta, x: A \vdash x: A & \frac{\Delta \vdash t: A_{i}}{\Delta \vdash \sigma_{i} t: A_{1}+A_{2}} \\
\Delta \vdash t: A_{1}+A_{2} \\
\frac{\Delta, x_{1}: A_{1} \vdash u_{1}: C \quad x_{2}: A_{2} \vdash u_{2}: C}{\Delta \vdash \delta\left(t, x_{1} \cdot u_{1}, x_{2} \cdot u_{2}\right): C}
\end{array}
$$

Figure 3. Typing rules for the simply-typed lambda-calculus

$$
\begin{gathered}
(\lambda x . t) u \rightarrow_{\beta} u[t / x] \quad(t: A \rightarrow B)={ }_{\eta} \lambda x . t x \\
\pi_{i}\left(t_{1}, t_{2}\right) \rightarrow_{\beta} t_{i} \quad(t: A * B)={ }_{\eta}\left(\pi_{1} t, \pi_{2} t\right) \\
\delta\left(\sigma_{i} t, x_{1} \cdot u_{1}, x_{2} . u_{2}\right) \rightarrow_{\beta} u_{i}\left[t / x_{i}\right] \\
\forall C[\square], \quad C[t: A+B]={ }_{\eta} \delta\left(t, x . C\left[\sigma_{1} x\right], x . C\left[\sigma_{2} x\right]\right)
\end{gathered}
$$

Figure 4. $\beta \eta$-equivalence for the simply-typed lambda-calculus

We distinguish positive types, negative types, and atomic types. The presentation of focusing (subsection 1.6) will justify this distinction. The equivalence rules of Figure 4 make it apparent that the $\eta$-equivalence rule for sums is more difficult to handle than the other $\eta$-rule, as it quantifies on any term context $C[\square]$. More generally, systems with only negative, or only positive types have an easier equational theory than those with mixed polarities. In fact, it is only at the end of the 20th century (Ghani 1995; Altenkirch, Dybjer, 
Hofmann, and Scott 2001; Balat, Di Cosmo, and Fiore 2004; Lindley 2007) that decision procedures for equivalence in the lambdacalculus with sums were first proposed.

Can we reduce the question of unicity to deciding equivalence? One would think of enumerating terms at the given type, and using an equivalence test as a post-processing filter to remove duplicates: as soon as one has found two distinct terms, the type can be declared non-uniquely inhabited. Unfortunately, this method does not give a terminating decision procedure, as naive proof search may enumerate infinitely many equivalent proofs, taking infinite time to post-process. We need to integrate canonicity in the structure of proof search itself.

\subsection{Terminology}

We distinguish and discuss the following properties:

- provability completeness: A search procedure is complete for provability if, for any type that is inhabited in the unrestricted type system, it finds at least one proof term.

- unicity completeness: A search procedure is complete for unicity if it is complete for provability and, if there exists two proofs distinct as programs in the unrestricted calculus, then the search finds at least two proofs distinct as programs.

- computational completeness: A search procedure is computationally complete if, for any proof term $t$ in the unrestricted calculus, there exists a proof in the restricted search space that is equivalent to $t$ as a program. This implies both previous notions of completeness.

- canonicity: A search procedure is canonical if it has no duplicates: any two enumerated proofs are distinct as programs. Such procedures require no filtering of results after the fact. We will say that a system is more canonical than another if it enumerates less redundant terms, but this does not imply canonicity.

There is a tension between computational completeness and termination of the corresponding search algorithm: when termination is obtained by cutting the search space, it may remove some computational behaviors. Canonicity is not a strong requirement: we could have a terminating, unicity-complete procedure and filter duplicates after the fact, but have found no such middle-ground. This article presents a logic that is both computationally complete and canonical (Section 3), and can be restricted (Section 4) to obtain a terminating yet unicity-complete algorithm (Section 5).

\subsection{Focusing for a less redundant proof search}

Focusing (Andreoli 1992) is a generic search discipline that can be used to restrict redundancy among searched proofs; it relies on the general idea that some proof steps are invertible (the premises are provable exactly when the conclusion is, hence performing this step during proof search can never lead you to a dead-end) while others are not. By imposing an order on the application of invertible and non-invertible proof steps, focusing restricts the number of valid proofs, but it remains complete for provability and, in fact, computationally complete $(\S 1.5)$.

More precisely, a focused proof system alternates between two phases of proof search. During the invertible phase, rules recognized as invertible are applied as long as possible - this stops when no invertible rule can be applied anymore. During the noninvertible phase, non-invertible rules are applied in the following way: a formula (in the context or the goal) is chosen as the focus, and non-invertible rules are applied as long as possible.

For example, consider the judgment $x: X+Y \vdash X+Y$. Introducing the sum on the right by starting with a $\sigma_{1}$ ? or $\sigma_{2}$ ? would be a non-invertible proof step: we are permanently committing to a choice - which would here lead to a dead-end. On the contrary, doing a case-split on the variable $x$ is an invertible step: it leaves all our options open. For non-focused proof search, simply using the variable $x: X+Y$ as an axiom would be a valid proof term. It is not a valid focused proof, however, as the case-split on $x$ is a possible invertible step, and invertible rules must be performed as long as they are possible. This gives a partial proof term $\delta(x, y . ?, z . ?)$, with two subgoals $y: X \vdash X+Y$ and $z: X \vdash X+Y$; for each of them, no invertible rule can be applied anymore, so one can only focus on the goal and do an injection. While the non-focused calculus had two syntactically distinct but equivalent proofs, $x$ and $\delta\left(x, y . \sigma_{1} y, z . \sigma_{2} z\right)$, only the latter is a valid focused proof: redundancy of proof search is reduced.

The interesting steps of a proof are the non-invertible ones. We call positive the type constructors that are "interesting to introduce". Conversely, their elimination rule is invertible (sums). We call negative the type constructors that are "interesting to eliminate", that is, whose introduction rule is invertible (arrow and product). While the mechanics of focusing are logic-agnostic, the polarity of constructors depends on the specific inference rules; linear logic needs to distinguish positive and negative products. Some focused systems also assign a polarity to atomic types, which allows to express interesting aspects of the dynamics of proof search (positive atoms correspond to forward search, and negative atoms to backward search). In Section 2 we present a simple focused variant of natural deduction for intuitionistic logic.

\subsection{Limitations of focusing}

In absence of sums, focused proof terms correspond exactly to $\beta$ short $\eta$-long normal forms. In particular, focused search is canonical $(\S 1.5)$. However, in presence of both polarities, focused proofs are not canonical anymore. They correspond to $\eta$-long form for the strictly weaker eta-rule defined without context quantification $x: A+B=_{\text {weak- } \eta} \delta\left(t, x . \sigma_{1} x, y . \sigma_{2} y\right)$.

This can be seen for example on the judgment $z: Z, x: Z \rightarrow$ $X+Y \vdash X+Y$, a variant on the previous example where the sum in the context is "thunked" under a negative datatype. The expected proof is $\delta\left(x z, y_{1} . \sigma_{1} y_{1}, y_{2} . \sigma_{2} y_{2}\right)$, but the focused discipline will accept infinitely many equivalent proof terms, such as $\delta\left(x z, y_{1} . \sigma_{1} y_{1}, y_{2} . \delta\left(x z, y_{1}^{\prime} . \sigma_{1} y_{1}^{\prime}, \ldots \sigma_{2} y_{2}\right)\right)$. The result of the application $x z$ can be matched upon again and again without breaking the focusing discipline.

This limitation can also be understood as a strength of focusing: despite equalizing more terms, the focusing discipline can still be used to reason about impure calculi where the eliminations corresponding to non-invertible proof terms may perform sideeffects, and thus cannot be reordered, duplicated or dropped. As we work on pure, terminating calculi - indeed, even adding nontermination as an uncontrolled effect ruins unicity - we need a stronger equational theory than suggested by focusing alone.

\subsection{Our idea: saturating proof search}

Our idea is that instead of only deconstructing the sums that appear immediately as the top type constructor of a type in context, we shall deconstruct all the sums that can be reached from the context by applying eliminations (function application and pair projection). Each time we introduce a new hypothesis in the context, we saturate it by computing all neutrals of sum type that can be built using this new hypothesis. At the end of each saturation phase, all the positives that could be deduced from the context have been deconstructed, and we can move forward applying non-invertible rules on the goal. Eliminating negatives until we get a positive and matching in the result corresponds to a cut (which is not reducible, as the scrutinee is a neutral term), hence our technique can be summarized as "Cut the positives as soon as you can". 
The idea was inspired by Sam Lindley's equivalence procedure for the lambda-calculus with sums, whose rewriting relation can be understood as moving case-splits down in the derivation tree, until they get blocked by the introduction of one of the variable appearing in their scrutinee (so moving down again would break scoping) - this also corresponds to "restriction (A)" in Balat, Di Cosmo, and Fiore (2004). In our saturating proof search, after introducing a new formal parameter in the context, we look for all possible new scrutinees using this parameter, and case-split on them. Of course, this is rather inefficient as most proofs will in fact not make use of the result of those case-splits, but this allows to give a common structure to all possible proofs of this judgment.

In our example $z: Z, x: Z \rightarrow X+Y \vdash X+Y$, the saturation discipline requires to cut on $x z$. But after this sum has been eliminated, the newly introduced variables $y_{1}: X$ or $y_{2}: Y$ do not allow to deduce new positives - we would need a new $Z$ for this. Thus, saturation stops and focused search restarts, to find a unique normal form $\delta\left(x z, y_{1} . \sigma_{1} y_{1}, y_{2} . \sigma_{2} y_{2}\right)$. In Section 3 we show that saturating proof search is computationally complete and canonical $(\S 1.5)$.

\subsection{Termination}

The saturation process described above does not necessarily terminate. For example, consider the type of Church numerals specialized to a positive $X+Y$, that is, $X+Y \rightarrow(X+Y \rightarrow X+Y) \rightarrow$ $X+Y$. Each time we cut on a new sum $X+Y$, we get new arguments to apply to the function $(X+Y \rightarrow X+Y)$, giving yet another sum to cut on.

In the literature on proof search for propositional logic, the usual termination argument is based on the subformula property: in a closed, fully cut-eliminated proof, the formulas that appear in subderivations of subderivations are always subformulas of the formulas of the main judgment. In particular, in a logic where judgments are of the form $S \vdash A$ where $S$ is a finite set of formulas, the number of distinct judgments appearing in subderivations is finite (there is a finite number of subformulas of the main judgment, and thus finitely many possible finite sets as contexts). Finally, in a goal-directed proof search process, we can kill any recursive subgoals whose judgment already appears in the path from the root of the proof to the subgoal. There is no point trying to complete a partial proof $P_{\text {above }}$ of $S \vdash A$ as a strict subproof of a partial proof $P_{\text {below }}$ of the same $S \vdash A$ (itself a subproof of the main judgment): if there is a closed subproof for $P_{\text {above }}$, we can use that subproof directly for $P_{\text {below }}$, obviating the need for proving $P_{\text {above }}$ in the first place. Because the space of judgments is finite, a search process forbidding such recurring judgments always terminates.

We cannot directly apply this reasoning, for two reasons.

- Our contexts are mapping from term variables to formulas or, seen abstractly, multisets of formulas; even if the space of possible formulas is finite for the same reason as above, the space of multisets over them is still infinite.

- Erasing such multiset to sets, and cutting according to the nonrecurrence criteria above, breaks unicity completeness $(\S 1.5)$. Consider the construction of Church numerals by a judgment of the form $x: X, y: X \rightarrow X \vdash X$. One proof is just $x$, and all other proofs require providing an argument of type $X$ to the function $y$, which corresponds to a subgoal that is equal to our goal; they would be forbidden by the no-recurrence discipline.

We must adapt these techniques to preserve not only provability completeness, but also unicity completeness $(\S 1.5)$. Our solution is to use bounded multisets to represent contexts and collect recursive subgoals. We store at most $M$ variables for each given formula, for a suitably chosen $M$ such that if there are two different programs for a given judgment $\Delta \vdash A$, then there are also two different programs for $\lfloor\Delta\rfloor_{M} \vdash A$, where $\lfloor\Delta\rfloor_{M}$ is the bounded erasure keeping at most $M$ variables at each formula.

While it seems reasonable that such a $M$ exists, it is not intuitively clear what its value is, or whether it is a constant or depends on the judgment to prove. Could it be that a given goal $A$ is provable in two different ways with four copies of $X$ in the context, but uniquely inhabited if we only have three $X$ ?

In Section 4 we prove that $M \stackrel{\text { def }}{=} 2$ suffices. In fact, we prove a stronger result: for any $n \in \mathbb{N}$, keeping at most $n$ copies of each formula in context suffices to find at least $n$ distinct proofs of any goal, if they exist.

For recursive subgoals as well, we only need to remember at most 2 copies of each subgoal: if some $P_{\text {above }}$ appears as the subgoal of $P_{\text {below }}$ and has the same judgment, we look for a closed proof of $P_{\text {above }}$. Because it would also have been a valid proof for $P_{\text {below }}$, we have found two proofs for $P_{\text {below }}$ the one using $P_{\text {above }}$ and its closed proof, and the closed proof directly. $P_{\text {above }}$ itself needs not allow new recursive subgoal at the same judgment, so we can kill any subgoal that has at least two ancestors with the same judgment while preserving completeness for unicity (§1.5).

\subsection{Contributions}

We show that the unique inhabitation problem for simply-typed lambda-calculus for sums is decidable, and propose an effective algorithm for it. Given a context and a type, it answers that there are zero, one, or "at least two" inhabitants, and correspondingly provides zero, one, or two distinct terms at this typing. Our algorithm relies on a novel saturating focused logic for intuitionistic natural deduction, with strong relations to the idea of maximal multi-focusing in the proof search literature (Chaudhuri, Miller, and Saurin 2008), that is both computationally complete ( $(1.5)$ and canonical with respect to $\beta \eta$-equivalence.

We provide an approximation result for program multiplicity of simply-typed derivations with bounded contexts. We use it to show that our terminating algorithm is complete for unicity $(\S 1.5)$, but it is a general result (on the common, non-focused intuitionistic logic) that is of independent interest.

Finally, we present preliminary studies of applications for code inference. While extension to more realistic type systems is left for future work, simply-typed lambda-calculus with atomic types already allow to encode some prenex-polymorphic types typically found in libraries of strongly-typed functional programs.

\section{Intuitionistic focused natural deduction}

In Figure 5 we introduce a focused natural deduction for intuitionistic logic, as a typing system for the simply-typed lambda-calculus - with an explicit let construct. It is relatively standard, strongly related to the linear intuitionistic calculus of Brock-Nannestad and Schürmann (2010), or the intuitionistic calculus of Krishnaswami (2009). We distinguish four judgments: $\Gamma ; \Delta \vdash_{\text {inv }} t: A$ is the invertible judgment, $\Gamma \vdash_{\text {foc }} t: P_{\text {at }}$ the focusing judgment, $\Gamma \vdash t \Uparrow A$ the non-invertible introduction judgment and $\Gamma \vdash n \Downarrow A$ the noninvertible elimination judgment. The system is best understood by following the "life cycle" of the proof search process (forgetting about proof terms for now), which initially starts with a sequent to prove of the form $\emptyset ; \Delta \vdash_{\text {inv }}$ ? : A.

During the invertible phase $\Gamma ; \Delta \vdash_{\text {inv }}$ ? : $A$, invertible rules are applied as long as possible. We defined negative types as those whose introduction in the goal is invertible, and positives as those whose elimination in the context is invertible. Thus, the invertible phase stops only when all types in the context are negative, and the goal is positive or atomic: this is enforced by the rule INV-END. The two contexts correspond to an "old" context $\Gamma$, which is negative or atomic (all positives have been eliminated in a previous invertible 


$$
\begin{aligned}
& \Gamma::=\operatorname{varmap}\left(N_{\mathrm{at}}\right) \quad \text { negative or atomic context } \\
& \Delta::=\operatorname{varmap}(A) \quad \text { general context } \\
& \text { INV-PAIR } \\
& \frac{\Gamma ; \Delta \vdash_{\text {inv }} t: A \quad \Gamma ; \Delta \vdash_{\text {inv }} u: B}{\Gamma ; \Delta \vdash_{\text {inv }}(t, u): A * B} \\
& \text { INV-SUM } \\
& \frac{\Gamma ; \Delta, x: A \vdash_{\mathrm{inv}} t: C \quad \Gamma ; \Delta, x: B \vdash_{\mathrm{inv}} u: C}{\Gamma ; \Delta, x: A+B \vdash_{\mathrm{inv}} \delta(x, x . t, x . u): C} \\
& \begin{array}{ll}
\text { INV-ARR } \\
\Gamma ; \Delta, x: A \vdash_{\text {inv }} t: B & \begin{array}{l}
\text { INV-END } \\
\Gamma ; \Delta \vdash_{\text {inv }} \lambda x . t: A \rightarrow B
\end{array} \quad \frac{\Gamma \Gamma^{\prime} \vdash_{\text {foc }} t: P_{\text {at }}}{\Gamma ; \Gamma^{\prime} \vdash_{\text {inv }} t: P_{\text {at }}}
\end{array} \\
& \text { FOC-INTRO FOC-ATOM } \\
& \frac{\Gamma \vdash t \Uparrow P}{\Gamma \vdash_{\text {foc }} t: P} \quad \frac{\Gamma \vdash n \Downarrow X}{\Gamma \vdash_{\text {foc }} n: X} \\
& \frac{\Gamma \vdash n \Downarrow P \quad \Gamma ; x: P \vdash_{\text {inv }} t: Q_{\text {at }}}{\Gamma \vdash_{\text {foc }} \text { let } x=n \text { in } t: Q_{\text {at }}} \quad \frac{\Gamma \vdash t \Uparrow A_{i}}{\Gamma \vdash \sigma_{i} t \Uparrow A_{1}+A_{2}} \\
& \begin{array}{lll}
\begin{array}{l}
\text { INTRO-END } \\
\Gamma ; \emptyset \vdash_{\text {inv }} t: N_{\text {at }} \\
\Gamma \vdash t \Uparrow N_{\text {at }}
\end{array} & \begin{array}{l}
\text { ELIM-PAIR } \\
\Gamma \vdash n \Downarrow A_{1} * A_{2}
\end{array} & \begin{array}{l}
\text { ELIM-START } \\
\Gamma \vdash \pi_{i} n \Downarrow A_{i}
\end{array}
\end{array} \\
& \frac{\stackrel{\text { ELIM-ARR }}{\Gamma \vdash n \Downarrow A \rightarrow B \quad \Gamma \vdash u \Uparrow A}}{\Gamma \vdash n u \Downarrow B}
\end{aligned}
$$

Figure 5. Cut-free focused natural deduction for intuitionistic logic

phase), and a "new" context $\Delta$ of any polarity, which is the one being processed by invertible rule. INV-END only applies when the new context $\Gamma^{\prime}$ is negative or atomic, and the goal $P_{\text {at }}$ positive or atomic.

The focusing phase $\Gamma \vdash_{\text {foc }} ?: P_{\text {at }}$ is where choices are made: a sequence of non-invertible steps will be started, and continue as long as possible. Those non-invertible steps may be eliminations in the context (FOC-ELIM), introductions of a strict positive in the goal (FOC-INTRO), or conclusion of the proof when the goal is atomic (FOC-ATOM).

In terms of search process, the introduction judgment $\Gamma \vdash$ ? $A$ should be read from the bottom to the top, and the elimination judgment $\Gamma \vdash$ ? $\Downarrow A$ from the top to the bottom. Introductions correspond to backward reasoning (to prove $A_{1}+A_{2}$ it suffices to prove $A_{i}$ ); they must be applied as long as the goal is positive, to end on negatives or atoms (INTRO-END) where invertible search takes over. Eliminations correspond to forward reasoning (from the hypothesis $A_{1} * A_{2}$ we can deduce $A_{i}$ ) started from the context (ELIM-START); they must also be applied as long as possible, as they can only end in the rule FOC-ELIM on a strict positive, or in the rule FOC-ATOM on an atom.

Sequent-style left invertible rules The left-introduction rule for sums INV-SUM is sequent-style rather than in the expected natural deduction style: we only destruct variables found in the context, instead of allowing to destruct arbitrary expressions. We also shadow the matched variable, as we know we will never need the sum again.

Let-binding The proof-term let $x=n$ in $t$ used in the FOC-ELIM rule is not part of the syntax we gave for the simply-typed lambda-calculus in Section 1.4. Indeed, focusing re-introduces a restricted cut rule which does not exist in standard natural deduction.
We could write $t[n / x]$ instead, to get a proper $\lambda$-term - and indeed when we speak of focused proof term as $\lambda$-term this substitution is to be understood as implicit. We prefer the let syntax which better reflects the dynamics of the search it witnesses.

We call letexp $(t)$ the $\lambda$-term obtained by performing letexpansion (in depth) on $t$, defined by the only non-trivial case:

$$
\text { letexp }(\operatorname{let} x=n \text { in } t) \stackrel{\text { def }}{=} \operatorname{letexp}(t)[\operatorname{let} \exp (n) / x]
$$

Normality If we explained let $x=n$ in $t$ as syntactic sugar for $(\lambda x . t) n$, our proofs term would contain $\beta$-redexes. We prefer to explain them as a notation for the substitution $t[n / x]$, as it is then apparent that proof term for the focused logic are in $\beta$-normal form. Indeed, $x$ being of strictly positive type, it is necessarily a sum and is destructed in the immediately following invertible phase by a rule INV-SUM (which shadows the variable, never to be used again). As the terms corresponding to non-invertible introductions $\Gamma \vdash n \Downarrow P$ are all neutrals, the substitution creates a subterm of the form $\delta(n, x . t, x . u)$ with no new redex.

One can also check that proof terms for judgments that do not contain sums are in $\eta$-long normal form. For example, a subterm of type $A \rightarrow B$ is either type-checked by an invertible judgment $\Gamma ; \Delta \vdash_{\text {inv }} t: A \rightarrow B$ or an elimination judgment $\Gamma \vdash n \Downarrow$ $A \rightarrow B$. In the first case, the invertible judgment is either a sum elimination (excluded by hypothesis) or a function introduction $\lambda x . u$. In the second case, because an elimination phase can only end on a positive or atomic type, we know that immediately below is the elimination rule for arrows: it is applied to some argument, and $\eta$-expanding it would create a $\beta$-redex.

Fact 1. The focused intuitionistic logic is complete for provability. It is also computationally complete (§1.5).

\subsection{Invertible commuting conversions}

The invertible commuting conversion (or invertible commutative cuts) relation $\left(=_{\text {icc }}\right)$ expresses that, inside a given invertible phase, the ordering of invertible step does not matter.

$$
\begin{gathered}
\delta\left(t, x . \lambda y_{1} \cdot u_{1}, x \cdot \lambda y_{2} \cdot u_{2}\right)={ }_{\mathrm{icc}} \lambda y \cdot \delta\left(t, x \cdot u_{1}\left[y / y_{1}\right], x \cdot u_{2}\left[y / y_{2}\right]\right) \\
\delta\left(t, x \cdot\left(u_{1}, u_{2}\right), x \cdot\left(r_{1}, r_{2}\right)\right)={ }_{\mathrm{icc}} \\
\left(\delta\left(t, x \cdot u_{1}, x \cdot r_{1}\right), \delta\left(t, x \cdot u_{2}, x \cdot r_{2}\right)\right) \\
\delta\left(t, x . \delta\left(u, y \cdot r_{1}, y \cdot r_{1}^{\prime}\right), x . \delta\left(u, y \cdot r_{2}, y \cdot r_{2}^{\prime}\right)\right)={ }_{\mathrm{icc}} \\
\delta\left(u, y . \delta\left(t, x \cdot r_{1}, x \cdot r_{2}\right), x \cdot \delta\left(t, x \cdot r_{1}^{\prime}, x \cdot r_{2}^{\prime}\right)\right)
\end{gathered}
$$

This equivalence relation is easily decidable. We could do without it. We could force a specific operation order by restricting typing rules, typically by making $\Delta$ a list to enforce sum-elimination order, and requiring the goal $C$ of sum-eliminations to be positive or atomic to enforce an order between sum-eliminations and invertibles utintroductions. We could also provide more expressive syntactic forms (parallel multi-sums elimination (Altenkirch, Dybjer, Hofmann, and Scott 2001)) and normalize to this more canonical syntax. We prefer to make the non-determinism explicit in the specification. Our algorithm uses some implementation-defined order for proof search, it never has to compute $(=\mathrm{icc})$-convertibility.

Note that there are term calculi (Curien and Munch-Maccagnoni 2010) inspired from sequent-calculus, where commuting conversions naturally correspond to computational reductions, which would form better basis for studying normal forms than $\lambda$-terms. In the present work we wished to keep a term language resembling functional programs.

\section{A saturating focused system}

In this section, we introduce the novel saturating focused proof search, again as a term typing system that is both computationally 
complete $(\S 1.5)$ and canonical. It serves as a specification of our normal forms; our algorithm shall only search for a finite subspace of saturated proofs, while remaining unicity complete.

Saturated focusing logic is a variant of the previous focused natural deduction, where the focusing judgment $\Gamma \vdash_{\text {foc }} t: P_{\text {at }}$ is replaced by a saturating judgment $\Gamma ; \Gamma^{\prime} \vdash_{\text {sat }} t: P_{\text {at }}$. The system is presented in Figure 6; the rules for non-invertible elimination and introductions, and the invertible rules, are identical to the previous ones and have not been repeated.

(rules for $\Gamma \vdash t \Uparrow A$ and $\Gamma \vdash n \Downarrow A$ as in Figure 5)

(invertible rules, except INV-END, as in Figure 5)

\begin{tabular}{|c|c|c|}
\hline $\begin{array}{l}\text { SINV-END } \\
\Gamma ; \Gamma^{\prime} \vdash \vdash_{\text {sat }} t: P_{\text {at }}\end{array}$ & $\begin{array}{l}\text { SAT-INTRO } \\
\quad \Gamma \vdash t \Uparrow P\end{array}$ & $\begin{array}{l}\text { SAT-ATOM } \\
\quad \Gamma \vdash n \Downarrow X\end{array}$ \\
\hline$\overline{\Gamma ; \Gamma^{\prime} \vdash_{\operatorname{sinv}} t: P_{\mathrm{at}}}$ & $\overline{\Gamma ; \emptyset \vdash_{\text {sat }} t: P}$ & $\overline{\Gamma ; \emptyset \vdash_{\text {sat }} n: X}$ \\
\hline \multicolumn{3}{|c|}{$\begin{array}{l}\mathrm{SAT} \\
(\bar{n}, \bar{P}) \subseteq\left\{(n, P) \mid\left(\Gamma, \Gamma^{\prime} \vdash n \Downarrow P\right) \wedge n \text { uses } \Gamma^{\prime}\right\} \\
\quad \Gamma, \Gamma^{\prime} ; \bar{x}: \bar{P} \vdash_{\text {sinv }} t: Q_{\text {at }} \quad \forall x \in \bar{x}, t \text { uses } x\end{array}$} \\
\hline \multicolumn{3}{|c|}{$\Gamma ; \Gamma^{\prime} \vdash_{\text {sat }}$ let $\bar{x}=\bar{n}$ in $t: Q_{\text {at }}$} \\
\hline$x \in \Delta$ & \multicolumn{2}{|c|}{$(\exists n \in \bar{n}, n$ uses $\Delta) \vee t$ uses $\Delta$} \\
\hline$\overline{x \text { uses } \Delta}$ & \multicolumn{2}{|c|}{ let $\bar{x}=\bar{n}$ in $t$ uses $\Delta$} \\
\hline \multicolumn{2}{|c|}{$\left(t_{1}\right.$ uses $\left.\Delta\right) \vee\left(t_{2}\right.$ uses $\left.\Delta\right)$} & $\vee(u$ uses $\Delta)$ \\
\hline \multicolumn{2}{|c|}{$\delta\left(x, x . t_{1}, x . t_{2}\right)$ uses $\Delta$} & uses $\Delta$ \\
\hline
\end{tabular}

Figure 6. Cut-free saturating focused intuitionistic logic

In this new judgment, the information that a part of the context is "new", which is available from the invertible judgment $\Gamma ; \Gamma^{\prime} \vdash_{\text {sinv }}$ $t: A$, is retained. The "old" context $\Gamma$ has already been saturated, and all the positives deducible from it have already been cut - the result of their destruction is somewhere in the context. In the new saturation phase, we must cut all new sums, that were not available before, that is, those that use $\Gamma^{\prime}$ in some way. It would not only be inefficient to cut old sums again, it would break canonicity ( $§ 1.5)$ : with redundant formal variables in the context our algorithm could wrongly believe to have found several distinct proofs.

The right-focusing rules SAT-INTRO and SAT-ATOM behave exactly as FOC-INTRO and FOC-ATOM in the previous focused system. But they can only be used when there is no new context.

When there is a new context to saturate, the judgment must go through the SAT rule - there is no other way to end the proof. The left premise of the rule, corresponding to the definition in SAT, quantifies over all strictly positive neutrals that can be deduced from the old and new contexts combined $\left(\Gamma, \Gamma^{\prime}\right)$, but selects those that are "new", in the sense that they use at least one variable coming from the new context fragment $\Gamma^{\prime}$. Then, we simultaneously cut on all those new neutrals, by adding a fresh variable for each of them in the general context, and continuing with an invertible phase: those positives need to be deconstructed for saturation to start again.

The $n$ uses $\Gamma^{\prime}$ restriction imposes a unique place at which each cut, each binder may be introduced in the proof term: exactly as soon as it becomes defineable. This enforces canonicity by eliminating redudant proofs that just differ in the place of introduction of a binder, or bind the same value twice. For example, consider the context $\Gamma \stackrel{\text { def }}{=}(x: X, y: X \rightarrow(Y+Y))$, and suppose we are tryind to find all distinct terms of type $Y$. During the first saturation phase $\left(\emptyset ; \Gamma \vdash_{\text {sat }}\right.$ ? : $\left.Y\right)$, we would build the neutral term $y x$ of type $Y+Y$; it passes the test $y x$ uses $\Gamma$ as it uses both variables of $\Gamma$. Then, the invertible phase $\Gamma ; z: Y+Y \vdash_{\operatorname{sinv}} ?: Y$ decomposes the goal in two subgoals $\Gamma ; z: Y \vdash_{\text {sat }} ?: Y$. Without the $n$ uses $\Gamma^{\prime}$ restriction, the SAT rule could cut again on $y x$, with would lead, after the next invertible phase, to contexts of the form $\Gamma, z: Y ; z^{\prime}: Y$. But it is wrong to have two distinct variables of type $Y$ here, as there should be only one way to build a $Y$.

The relation $n$ uses $\Gamma^{\prime}$ is defined structurally on proof terms (or, equivalently, their typing derivations). Basically, a term "uses" a context if it uses at least one of its variables; for most terms, it is defined as a big "or" on its subterms. The only subtlety is that the case-split $\delta\left(x, x . t_{1}, x . t_{2}\right)$ does not by itself count as a use of the split variable: to be counted as "used", either $t_{1}$ or $t_{2}$ must use the shadowing variable $x$.

Finally, the last condition of the SAT rule $(\forall x \in \bar{x}, t$ uses $x)$ restricts the saturated variables listed in the let-binding to be only those actually used by the term. In terms of proof search, this restriction is applied after the fact: first, cut all positives, then search for all possible subproofs, and finally trim each of them, so that it binds only the positives it uses. This restriction thus does not influence proof search, but it ensures that there always exist finite saturating proofs for inhabited types, by allowing proof search to drop unnecessary bindings instead of saturating them forever. Consider Church numerals on a sum type, $X+Y \rightarrow(X+Y \rightarrow$ $X+Y) \rightarrow X+Y$, there would be no finite saturating proof without this restriction, which would break provability completeness.

Theorem 1 (Canonicity of saturating focused logic). If we have $\Gamma ; \Delta \vdash_{\operatorname{sinv}} t: A$ and $\Gamma ; \Delta \vdash_{\operatorname{sinv}} u: A$ in saturating focused logic with $t \neq_{\text {icc }} u$, then $t \neq_{\beta \eta} u$.

Theorem 2 (Computational completeness of saturating focused logic). If we have $\emptyset ; \Delta \vdash_{\text {inv }} t: A$ in the non-saturating focused logic, then for some $u={ }_{\beta \eta} t$ we have $\emptyset ; \Delta \vdash_{\sin v} u: A$ in the saturating focused logic.

\section{Two-or-more approximation}

A complete presentation of the content of this section, along with complete proofs, is available as a research report (Scherer 2014).

Our algorithm bounds contexts to at most two formal variables at each type. To ensure it correctly predicts unicity (it never claims that there are zero or one programs when two distinct programs exist), we need to prove that if there exists two distinct saturated proofs of a goal $A$ in a given context $\Gamma$, then there already exist two distinct proofs of $A$ in the context $[\Gamma\rfloor_{2}$, which drops variables from $\Gamma$ so that no formula occurs more than twice.

We formulate this property in a more general way: instead of talking about the cut-free proofs of the saturating focused logics, we prove a general result about the set of derivations of a typing judgment $\Delta \vdash ?: A$ that have "the same shape", that is, that erase to the same derivation of intuitionistic logic $\lfloor\Delta\rfloor_{1} \vdash A$, where $\lfloor\Delta\rfloor_{1}$ is the set of formulas present in $\Delta$, forgetting multiplicity. This result applies in particular to saturating focused proof terms, (their let-expansion) seen as programs in the unfocused $\lambda$-calculus.

We define an explicit syntax for "shapes" $S$ in Figure 7, which are in one-to-one correspondence with (variable-less) natural deduction proofs. It also define the erasure function $\lfloor t\rfloor_{1}$ from typed $\lambda$-terms to typed shapes.

The central idea of our approximation result is the use of counting logics, that counts the number of $\lambda$-terms of different shapes. A counting logic is parametrized over a semiring ${ }^{1} K$; picking the

\footnotetext{
${ }^{1}$ A semiring $\left(K, 0_{K}, 1_{K},+_{K}, \times_{K}\right)$ is defined as a two-operation algebraic structure where $\left(0_{K},+_{K}\right)$ and $\left(1_{K}, \times_{K}\right)$ are monoids, $\left(+_{K}\right)$ commutes and distributes over $\left(\times_{K}\right)$ (which may or may not commute), $0_{K}$ is a zero/absorbing element for $\left(\times_{K}\right)$, but $\left(+_{K}\right)$ and $\left(\times_{K}\right)$ need not have inverses ( $\mathbb{Z}$ 's addition is invertible so it is a ring, $\mathbb{N}$ is only a semiring).
} 


$$
\begin{aligned}
& S, T \quad:= \\
& A, B, C, D \\
& \lambda A . S \\
& S T \\
& (S, T) \\
& \pi_{i} S \\
& \sigma_{i} S \\
& \delta\left(S, A . T_{1}, B . T_{2}\right) \text { sum destruction } \\
& \lfloor x: A\rfloor_{1} \stackrel{\text { def }}{=} A \quad\lfloor\lambda x: A . t\rfloor_{1} \stackrel{\text { def }}{=} \lambda A .\lfloor t\rfloor_{1} \\
& \lfloor t u\rfloor_{1} \stackrel{\text { def }}{=}\lfloor t\rfloor_{1}\lfloor u\rfloor_{1} \quad\lfloor(t, u)\rfloor_{1} \stackrel{\text { def }}{=}\left(\lfloor t\rfloor_{1},\lfloor u\rfloor_{1}\right) \\
& \left\lfloor\pi_{i} t\right\rfloor_{1} \stackrel{\text { def }}{=} \pi_{i}\lfloor t\rfloor_{1} \quad\left\lfloor\sigma_{i} t\right\rfloor_{1} \stackrel{\text { def }}{=} \sigma_{i}\lfloor t\rfloor_{1} \\
& \lfloor\delta((t: A+B), y \cdot u, z \cdot r)\rfloor_{1} \stackrel{\text { def }}{=} \delta\left(\lfloor t\rfloor_{1}, A \cdot\lfloor u\rfloor_{1}, B \cdot\lfloor r\rfloor_{1}\right)
\end{aligned}
$$

Figure 7. Shapes of variable-less natural deduction proofs

semiring of natural numbers precisely corresponds to counting the number of terms of a given shape, counting in the semiring $\{(0,1)\}$ corresponds to the variable-less logic (which only expresses inhabitation), and counting in finite semirings of support $\{0,1, \ldots, M\}$ corresponds to counting proofs with approximative bounded contexts of size at most $M$.

The counting logic, defined in Figure 8, is parametrized over a semiring $\left(K, 0_{K}, 1_{K},+_{K}, \times_{K}\right)$. The judgment is of the form $S:: \Phi \vdash_{K} A: a$, where $S$ is the shape of corresponding logic derivation, $\Phi$ is a context mapping formulas to a multiplicity in $K$, $A$ is the type of the goal being proven, and $a$ is the "output count", a scalar of $K$.

$$
\begin{aligned}
&(\Phi, \Psi) \stackrel{\text { def }}{=} A \mapsto\left(\Phi(A)+_{K} \Psi(A)\right) \\
&(A: 1) \stackrel{\text { def }}{=} \begin{cases}A & \mapsto 1_{K} \\
B \neq A & \mapsto 0_{K}\end{cases}
\end{aligned}
$$

$$
\begin{array}{ll}
\text { COUNT-AXIOM } & \text { COUnT-INTRO-ARR } \\
A:: \Phi \vdash_{K} A: \Phi(A) & \frac{S:: \Phi, A: 1 \vdash_{K} B: a}{\lambda A . S:: \Phi \vdash_{K} A \rightarrow B: a}
\end{array}
$$

COUNT-ELIM-ARR

$$
\frac{S_{1}:: \Phi \vdash_{K} A \rightarrow B: a_{1} \quad S_{2}:: \Phi \vdash_{K} A: a_{2}}{S_{1} S_{2}:: \Phi \vdash_{K} B: a_{1} \times a_{2}}
$$

$$
\begin{aligned}
& \text { COUnT-INTRO-PAIR } \\
& \frac{S_{1}:: \Phi \vdash_{K} A: a_{1} \quad S_{2}:: \Phi \vdash_{K} B: a_{2}}{\left(S_{1}, S_{2}\right):: \Phi \vdash_{K} A * B: a_{1} \times a_{2}}
\end{aligned}
$$

$$
\begin{aligned}
& \text { COUNT-ELIM-PAIR } \\
& \frac{S:: \Phi \vdash_{K} A_{1} * A_{2}: a}{\pi_{i} S:: \Phi \vdash_{K} A_{i}: a} \\
& \text { COUNT-INTRO-SUM } \\
& \frac{S:: \Phi \vdash_{K} A_{i}: a}{\sigma_{i} S:: \Phi \vdash_{K} A_{1}+A_{2}: a} \\
& \text { COUNT-ELIM-SUM } \\
& S:: \Phi \vdash_{K} A+B: a_{1} \\
& \frac{T_{1}:: \Phi, A: 1 \vdash_{K} C: a_{2} \quad T_{2}:: \Phi, B: 1 \vdash_{K} C: a_{3}}{\delta\left(S, A . T_{1}, B . T_{2}\right):: \Phi \vdash_{K} C: a_{1} \times a_{2} \times a_{3}}
\end{aligned}
$$

Figure 8. Counting logic over $\left(K, 0_{K}, 1_{K},+_{K}, \times_{K}\right)$

Let us write \#S the cardinal of a set $S$ and $\lfloor\Delta\rfloor_{\#}$ for the "cardinal erasure" of the typing context $\Delta$, defined as $\#\{x \mid(x$ : A) $\in \Delta\}$. We can express the relation between counts in the semiring $\mathbb{N}$ and cardinality of typed $\lambda$-terms of a given shape:
Lemma 1. For any environment $\Delta$, shape $S$ and type $A$, the following counting judgment is derivable:

$$
S::\lfloor\Delta\rfloor_{\#} \vdash_{\mathbb{N}} A: \#\left\{t \mid \Delta \vdash t: A \wedge\lfloor t\rfloor_{1}=S\right\}
$$

Note that the counting logic does not have a convincing dynamic semantics - the dynamic semantics of variable-less shapes themselves have been studied in Dowek and Jiang (2011). We only use it as a reasoning tool to count programs.

If $\phi: K \rightarrow K^{\prime}$ map the scalars of one semiring to another, and $\Phi$ is a counting context in $K$, we write $\lfloor\Phi\rfloor_{\phi}$ its erasure in $K^{\prime}$ defined by $\lfloor\Phi\rfloor_{\phi}(A) \stackrel{\text { def }}{=} \phi(\Phi(A))$. We can then formulate the main result on counting logics:

Theorem 3 (Morphism of derivations). If $\phi: K \rightarrow K^{\prime}$ is a semiring morphism and $S:: \Phi \vdash_{K} A: a$ is derivable, then $S::\lfloor\Phi\rfloor_{\phi} \vdash_{K^{\prime}} A: \phi(a)$ is also derivable.

To conclude, we only need to remark that the derivation count is uniquely determined by the multiplicity context.

Lemma 2 (Determinism). If we have both $S:: \Phi \vdash_{K} A:$ a and $S:: \Phi \vdash_{K} A: b$ then $a={ }_{K} b$.

Corollary 1 (Counting approximation). If $\phi$ is a semiring morphism and $\lfloor\Phi\rfloor_{\phi}=\lfloor\Psi\rfloor_{\phi}$ then $S:: \Phi \vdash_{K} A:$ a and $S:: \Psi \vdash_{K}$ $A:$ imply $\phi(a)=\phi(b)$.

Approximating arbitrary contexts into zero, one or "two-ormore" variables corresponds to the semiring $\overline{2}$ of support $\{0,1,2\}$, with commutative semiring operations fully determined by $1+1=$ $2,2+a=2$, and $2 \times 2=2$. Then, the function $n \mapsto \min (2, n)$ is a semiring morphism from $\mathbb{N}$ to $\overline{2}$, and the corollary above tells us that number of derivations of the judgments $\Delta \vdash A$ and $\lfloor\Delta\rfloor_{2} \vdash A$ project to the same value in $\{0,1,2\}$. This results extend to any $n$, as $\{0,1, \ldots, n\}$ can be similarly given a semiring structure.

\section{Search algorithm}

The saturating focused logic corresponds to a computationally complete presentation of the structure of canonical proofs we are interested in. From this presentation it is extremely easy to derive a terminating search algorithm complete for unicity - we moved from a whiteboard description of the saturating rules to a working implementation of the algorithm usable on actual examples in exactly one day of work. The implementation (Scherer and Rémy 2015) is around 700 lines of readable OCaml code.

The central idea to cut the search space while remaining complete for unicity is the two-or-more approximation: there is no need to store more than two formal variables of each type, as it suffices to find at least two distinct proofs if they exist - this was proved in the Section 4. We use a plurality monad Plur, defined in set-theoretic terms as $\operatorname{Plur}(S) \stackrel{\text { def }}{=} 1+S+S \times S$, representing zero, one or "at least two" distinct elements of the set $S$. Each typing judgment is reformulated into a search function which takes as input the context(s) of the judgment and its goal, and returns a plurality of proof terms - we search not for one proof term, but for (a bounded set of) all proof terms. Reversing the usual mapping from variables to types, the contexts map types to pluralities of formal variables.

In the search algorithm, the SINV-END rule does not merely pass its new context $\Gamma^{\prime}$ to the saturation rules, but it also trims it by applying the two-or-more rule: if the old context $\Gamma$ already has two variables of a given formula $N_{\text {at }}$, drop all variables for $N_{\text {at }}$ from $\Gamma^{\prime}$; if it already has one variable, retain at most one variable in $\Gamma^{\prime}$. This corresponds to an eager application of the variable-use restriction of the SAT rule: we have decided to search only for terms that will not use those extraneous variables, hence they are never useful during saturation and we may as well drop them now. This 
trimming is sound, because it corresponds to an application of the SAT rule that would bind the empty set. Proving that it is complete for unicity is the topic of Section 4.

To effectively implement the saturation rules, a useful tool is a selection function (called select_oblis in our prototype) which takes a selection predicate on positive or atomic formulas $P_{\mathrm{at}}$, and selects (a plurality of) each negative formula $N_{\text {at }}$ from the context that might be the starting point of an elimination judgment of the form $\Gamma \vdash n \Downarrow P_{\text {at }}$, for a $P_{\text {at }}$ accepted by the selection predicate. For example, if we want to prove $X$ and there is a formula $Y \rightarrow Z * X$, this formula will be selected - although we don't know yet if we will be able to prove $Y$. For each such $P_{\text {at }}$, it returns a proof obligation, that is either a valid derivation of $\Gamma \vdash n \Downarrow P_{\text {at }}$, or a request, giving some formula $A$ and expecting a derivation of $\Gamma \vdash ? \Uparrow A$ before returning another proof obligation.

The rule SAT-Атом $\left(\Gamma ; \emptyset \vdash_{\text {sat }} ?: X\right)$ uses this selection function to select all negatives that could potentially be eliminated into a $X$, and feeding (pluralities of) answers to the returned proof obligations (by recursively searching for introduction judgments) to obtain (pluralities of) elimination proofs of $X$.

The rule SAT uses the selection function to find the negatives that could be eliminated in any strictly positive formula and tries to fullfill (pluralities of) proof obligations. This returns a binding context (with a plurality of neutrals for each positive formula), which is filtered a posteriori to keep only the "new" bindings that use the new context. The new binding are all added to the search environment, and saturating search is called recursively. It returns a plurality of proof terms; each of them results in a proof derivation (where the saturating set is trimmed to retain only the bindings useful to that particular proof term).

Finally, to ensure termination while remaining complete for unicity, we do not search for proofs where a given subgoal occurs strictly more than twice along a given search path. This is easily implemented by threading an extra "memory" argument through each recursive call, which counts the number of identical subgoals below a recursive call and kills the search (by returning the "zero" element of the plurality monad) at two. Note that this does not correspond to memoization in the usual sense, as information is only propagated along a recursive search branch, and never shared between several branches.

This fully describes the algorithm, which is easily derived from the logic. It is effective, and our implementation answers instantly on all the (small) types of polymorphic functions we tried. But it is not designed for efficiency, and in particular saturation duplicates a lot of work (re-computing old values before throwing them away).

In Appendix A, we give a presentation of the algorithm as a system of inference rules that is terminating and deterministic. Using the two-or-more counting approximation result (Corollary 1) of the next section, we can prove the correctness of this presentation.

Theorem 4. Our unicity-deciding algorithm is terminating and complete for unicity.

The search space restrictions described above are those necessary for termination. Many extra optimizations are possible, that can be adapted from the proof search literature - with some care to avoid losing completness for unicity. For example, there is no need to cut on a positive if its atoms do not appear in negative positions (nested to the left of an odd number of times) in the rest of the goal. We did not develop such optimizations, except for two low-hanging fruits we describe below.

Eager redundancy elimination Whenever we consider selecting a proof obligation to prove a strict positive during the saturation phase, we can look at the negatives that will be obtained by cutting it. If all those atoms are already present at least twice in the context, this positive is redundant and there is no need to cut on it. Dually, before starting a saturation phase, we can look at whether it is already possible to get two distinct neutral proofs of the goal from the current context. In this case it is not necessary to saturate at all.

This optimization is interesting because it significantly reduces the redundancy implied by only filtering of old terms after computing all of them. Indeed, we intuitively expect that most types present in the context are in fact present twice (being unique tends to be the exception rather than the rule in programming situations), and thus would not need to be saturated again. Redundancy of saturation still happens, but only on the "frontier formulas" that are present exactly once.

Subsumption by memoization One of the techniques necessary to make the inverse method (McLaughlin and Pfenning 2008) competitive is subsumption: when a new judgment is derived by forward search, it is added to the set of known results if it is not subsumed by a more general judgment (same goal, smaller context) already known.

In our setting, being careful not to break computational completeness, this rule becomes the following. We use (monotonic) mutable state to grow a memoization table of each proved subgoal, indexed by the right-hand-side formula. Before proving a new subgoal, we look for all already-computed subgoals of the same righthand-side formula. If one exists with exactly the same context, we return its result. But we also return eagerly if there exists a larger context (for inclusion) that returned zero result, or a smaller context that returned two-or-more results.

Interestingly, we found out that this optimization becomes unsound in presence of the empty type 0 (which are not yet part of the theory, but are present as an experiment in our implementation). Its equational theory tells us that in an inconsistent cotnext ( 0 is provable), all proofs are equal. Thus a type may have two inhabitants in a given context, but a larger context that is inconsistent (allows to prove 0 ) will have a unique inhabitant, breaking monotonicity.

\section{Evaluation}

In this section, we give some practical examples of code inference scenarios that our current algorithm can solve, and some that it cannot - because the simply-typed theory is too restrictive.

The key to our application is to translate a type using prenexpolymorphism into a simple type using atoms in stead of type variables - this is semantically correct given that bound type variables in System F are handled exactly as simply-typed atoms. The approach, of course, is only a very first step and quickly shows it limits. For example, we cannot work with polymorphic types in the environment (ML programs typically do this, for example when typing a parametrized module, or type-checking under a type-class constraint with polymorphic methods), or first-class polymorphism in function arguments. We also do not handle higher-kinded types - even pure constructors.

\subsection{Inferring polymorphic library functions}

The Haskell standard library contains a fair number of polymorphic functions with unique types. The following examples have been checked to be uniquely defined by their types:

$$
\begin{gathered}
\text { fst }: \forall \alpha \beta . \alpha * \beta \rightarrow \alpha \\
\text { curry : } \forall \alpha \beta \gamma \cdot(\alpha * \beta \rightarrow \gamma) \rightarrow \alpha \rightarrow \beta \rightarrow \gamma \\
\text { uncurry : } \forall \alpha \beta \gamma .(\alpha \rightarrow \beta \rightarrow \gamma) \rightarrow \alpha * \beta \rightarrow \gamma \\
\text { either }: \forall \alpha \beta \gamma \cdot(\alpha \rightarrow \gamma) \rightarrow(\beta \rightarrow \gamma) \rightarrow \alpha+\beta \rightarrow \gamma
\end{gathered}
$$

When the API gets more complicated, both types and terms become harder to read and uniqueness of inhabitation gets much less obvious. Consider the following operators chosen arbitrarily in the lens (Kmett 2012) library. 


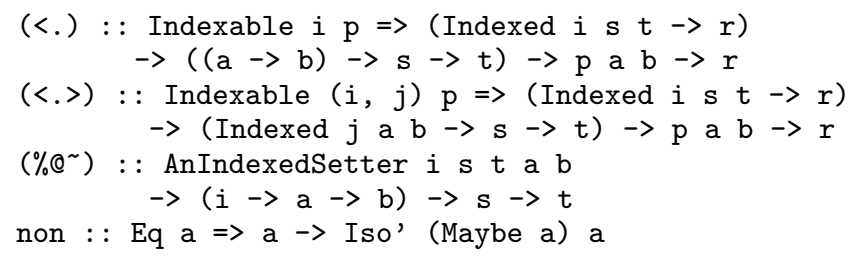

The type and type-class definitions involved in this library usually contain first-class polymorphism, but the documentation (Kmett 2013) provides equivalent "simple types" to help user understanding. We translated the definitions of Indexed, Indexable and Iso using those simple types. We can then check that the first three operators are unique inhabitants; non is not.

\subsection{Inferring module implementations or type-class instances}

The Arrow type-class is defined as follows:

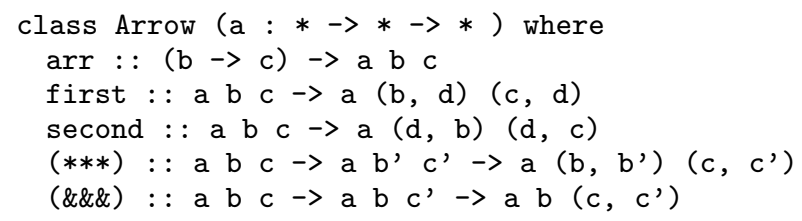

It is self-evident that the arrow type $(\rightarrow)$ is an instance of this class, and no code should have to be written to justify this: our prototype is able to infer that all those required methods are uniquely determined when the type constructor a is instantiated with an arrow type. This also extends to subsequent type-classes, such as ArrowChoice.

As most of the difficulty in inferring unique inhabitants lies in sums, we study the "exception monad", that is, for a fixed type $X$, the functor $\alpha \mapsto X+\alpha$. Our implementation determines that its Functor and Monad instances are uniquely determined, but that its Applicative instance is not.

Indeed, the type of the Applicative method ap specializes to the following: $\forall \alpha \beta . X+(\alpha \rightarrow \beta) \rightarrow X+\alpha \rightarrow X+\beta$. If both the first and the second arguments are in the error case $X$, there is a non-unique choice of which error to return in the result.

This is in fact a general result on applicative functors for types that are also monads: there are two distinct ways to prove that a monad is also an applicative functor.

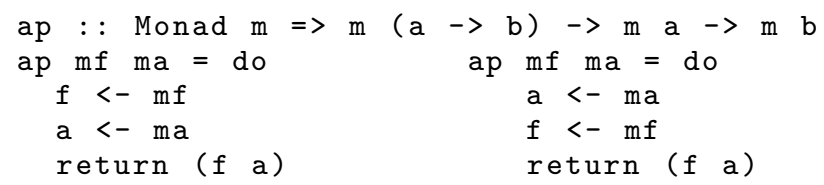

Note that the type of bind for the exception monad, namely $\forall \alpha \beta . X+\alpha \rightarrow(\alpha \rightarrow X+\beta) \rightarrow X+\beta$, has a sum type thunked under a negative type. It is one typical example of type which cannot be proved unique by the focusing discipline alone, which is correctly recognized unique by our algorithm.

\subsection{Non-applications}

Here are two related ideas we wanted to try, but that do not fit in the simply-typed lambda-calculus; the uniqueness algorithm must be extended to richer type systems to handle such applications.

We can check that specific instances of a given type-class are canonically defined, but it would be nice to show as well that some of the operators defined on any instance are uniquely defined from the type-class methods - although one would expect this to often fail in practice if the uniqueness checker doesn't understand the equational laws required of valid instances. Unfortunately, this would require uniqueness check with polymorphic types in context (for the polymorphic methods).
Another idea is to verify the coherence property of a set of declared instances by translating instance declarations into terms, and checking uniqueness of the required instance types. In particular, one can model the inheritance of one class upon another using a pair type (Comp $\alpha$ as a pair of a value of type $\mathrm{Eq} \alpha$ and Compspecific methods); and the system can then check that when an instance of Eq $X$ and Comp $X$ are declared, building Eq $X$ directly or projecting it from Comp $X$ correspond to $\beta \eta$-equivalent elaboration witnesses. Unfortunately, all but the most simplistic examples require parametrized types and polymorphic values in the environment to be faithfully modelled.

\subsection{On impure host programs}

The type system in which program search is performed does not need to exactly coincide with the ambiant type system of the host programming language, for which the code-inference feature is proposed - forcing the same type-system would kill any use from a language with non-termination as an effect. Besides doing term search in a pure, terminating fragment of the host language, one could also refine search with type annotations in a richer type system, eg. using dependent types or substructural logic - as long as the found inhabitants can be erased back to host types.

However, this raises the delicate question of, among the unique $\beta \eta$-equivalence class of programs, which candidate to select to be actually injected into the host language. For example, the ordering or repetition of function calls can be observed in a host language passing impure function as arguments, and $\eta$-expansion of functions can delay effects. Even in a pure language, $\eta$-expanding sums and products may make the code less efficient by re-allocating data. There is a design space here that we have not explored.

\section{Conclusion}

\subsection{Related work}

Previous work on unique inhabitation The problem of unique inhabitation for the simply-typed lambda-calculus (without sums) has been formulated by Mints (1981), with early results by Babaev and Solov'ev (1982), and later results by Aoto and Ono (1994); Aoto (1999) and Broda and Damas (2005).

These works have obtained several different sufficient conditions for a given type to be uniquely inhabited. While these cannot be used as an algorithm to decide unique inhabitation for any type, it reveals fascinating connections between unique inhabitation and proof or term structures. Some sufficient criterions are formulated on the types/formulas themselves, other on terms (a type is uniquely inhabited if it is inhabited by a term of a given structure).

A simple criterion on types given in Aoto and Ono (1994) is that "negatively non-duplicated formulas", that is formulas where each atom occurs at most once in negative position (nested to the left of an odd number of arrows), have at most one inhabitant. This was extended by Broda and Damas (2005) to a notion of "deterministic" formulas, defined using a specialized representation for simplytyped proofs named "proof trees".

Aoto (1999) proposed a criterion based on terms: a type is uniquely inhabited if it "provable without non-prime contraction", that is if it has at least one inhabitant (not necessarily cut-free) whose only variables with multiple uses are of atomic type. Recently, Bourreau and Salvati (2011) used game semantics to give an alternative presentation of Aoto's results, and a syntactic characterization of all inhabitants of negatively non-duplicated formulas.

Those sufficient conditions suggest deep relations between the static and dynamics semantics of restricted fragments of the lambda-calculus - it is not a coincidence that contraction at nonatomic type is also problematic in definitions of proof equivalence coming from categorial logic (Dosen 2003). However, they give 
little in the way of a decision procedure for all types - conversely, our decision procedure does not by itself reveal the structure of the types for which it finds unicity.

An indirectly related work is the work on retractions in simple types ( $A$ is a retract of $B$ if $B$ can be surjectively mapped into $A$ by a $\lambda$-term). Indeed, in a type system with a unit type 1 , a given type $A$ is uniquely inhabited if and only if it is a retract of 1 . Stirling (2013) proposes an algorithm, inspired by dialogue games, for deciding retraction in the lambda-calculus with arrows and products; but we do not know if this algorithm could be generalized to handle sums. If we remove sums, focusing already provides an algorithm for unique inhabitation.

Counting inhabitants Broda and Damas (2005) remark that normal inhabitants of simple types can be described by a context-free structure. This suggests, as done in Zaoinc (1995), counting terms by solving a set of polynomial equations. Further references to such "grammatical" approaches to lambda-term enumeration and counting can be found in Dowek and Jiang (2011).

Of particular interest to us was the recent work of Wells and Yakobowski (2004). It is similar to our work both in terms of expected application (program fragment synthesis) and methods, as it uses (a variant of) the focused calculus LJT (Herbelin 1993) to perform proof search. It has sums (disjunctions), but because it only relies on focusing for canonicity it only implements the weak notion of $\eta$-equivalence for sums: as explained in Section 1.7, it counts an infinite number of inhabitants in presence of a sum thunked under a negative. Their technique to ensure termination of enumeration is very elegant. Over the graph of all possible proof steps in the type system (using multisets as contexts: an infinite search space), they superimpose the graph of all possible noncyclic proof steps in the logic (using sets as contexts: a finite search space). Termination is obtained, in some sense, by traversing the two in lockstep. We took inspiration from this idea to obtain our termination technique: our bounded multisets can be seen as a generalization of their use of set-contexts.

Non-classical theorem proving and more canonical systems Automated theorem proving has motivated fundamental research on more canonical representations of proofs: by reducing the number of redundant representations that are equivalent as programs, one can reduce the search space - although that does not necessarily improve speed, if the finer representation requires more bookkeeping. Most of this work was done first for (first-order) classical logic; efforts porting them to other logics (linear, intuitionistic, modal) were of particular interest, as it often reveals the general idea behind particular techniques, and is sometimes an occasion to reformulate them in terms closer to type theory.

An important brand of work studies connection-based, or matrix-based, proof methods. They have been adapted to nonclassical logic as soon as Wallen (1987). It is possible to present connection-based search "uniformly" for many distinct logics (Otten and Kreitz 1996), changing only one logic-specific check to be performed a posteriori on connections (axiom rules) of proof candidates. In intuitionistic setting, that would be a comparison on indices of Kripke Worlds; it is strongly related to labeled logics (Galmiche and Méry 2013). On the other hand, matrix-based methods rely on guessing the number of duplications of a formula (contractions) that will be used in a particular proof, and we do not know whether that can be eventually extended to second-order polymorphism - by picking a presentation closer to the original logic, namely focused proofs, we hope for an easier extension.

Some contraction-free calculi have been developed with automated theorem proving for intuitionistic logic in mind. A presentation is given in Dyckhoff (1992) - the idea itself appeared as early as Vorob'ev (1958). The idea is that sums and (positive) prod- ucts do not need to be deconstructed twice, and thus need not be contracted on the left. For functions, it is actually sufficient for provability to implicitly duplicate the arrow in the argument case of its elimination form ( $A \rightarrow B$ may have to be used again to build the argument $A$ ), and to forget it after the result of application $(B)$ is obtained. More advanced systems typically do casedistinctions on the argument type $A$ to refine this idea, see Dyckhoff (2013) for a recent survey. Unfortunately, such techniques to reduce the search space break computational completeness: they completely remove some programmatic behaviors. Consider the type $\operatorname{Stream}(A, B) \stackrel{\text { def }}{=} A *(A \rightarrow A * B)$ of infinite streams of state $A$ and elements $B$ : with this restriction, the next-element function can be applied at most once, hence $\operatorname{Stream}(X, Y) \rightarrow Y$ is uniquely inhabited in those contraction-free calculi. (With focusing, only negatives are contracted, and only when picking a focus.)

Focusing was introduced for linear logic (Andreoli 1992), but is adaptable to many other logics. For a reference on focusing for intuitionistic logic, see Liang and Miller (2007). To easily elaborate programs as lambda-terms, we use a natural deduction presentation (instead of the more common sequent-calculus presentation) of focused logic, closely inspired by the work of Brock-Nannestad and Schürmann (2010) on intuitionistic linear logic.

Some of the most promising work on automated theorem proving for intuitionistic logic comes from applying the so-called "Inverse Method" (see Degtyarev and Voronkov (2001) for a classical presentation) to focused logics. The inverse method was ported to linear logic in Chaudhuri and Pfenning (2005), and turned into an efficient implementation of proof search for intuitionistic logic in McLaughlin and Pfenning (2008). It is a "forward" method: to prove a given judgment, start with the instances of axiom rules for all atoms in the judgment, then build all possible valid proofs until the desired judgment is reached - the subformula property, bounding the search space, ensures completeness for propositional logic. Focusing allows important optimization of the method, notably through the idea of "synthetic connectives": invertible or noninvertible phases have to be applied all in one go, and thus form macro-steps that speed up saturation.

In comparison, our own search process alternates forward and backward-search. At a large scale we do a backward-directed proof search, but each non-invertible phase performs saturation, that is a complete forward-search for positives. Note that the search space of those saturation phases is not the subformula space of the main judgment to prove, but the (smaller) subformula space of the current subgoal's context. When saturation is complete, backward goal-directed search restarts, and the invertible phase may grow the context, incrementally widening the search space. (The forwarddirected aspects of our system could be made richer by adding positive products and positively-biased atoms; this is not our main point of interest here. Our coarse choice has the good property that, in absence of sum types in the main judgment, our algorithm immediately degrades to simple, standard focused backward search.)

Lollimon (López, Pfenning, Polakow, and Watkins 2005) mixes backward search for negatives and forward search for positives. The logic allows but does not enforce saturation; it is only in the implementation that (provability) saturation is used, and they found it useful for their applications - modelling concurrent systems.

Finally, an important result for canonical proof structures is maximal multi-focusing (Miller and Saurin 2007; Chaudhuri, Miller, and Saurin 2008). Multi-focusing refines focusing by introducing the ability to focus on several formulas at once, in parallel, and suggests that, among formulas equivalent modulo valid permutations of inference rules, the "more parallel" one are more canonical. Indeed, maximal multi-focused proofs turn out to be equivalent to existing more-canonical proof structures such as linear proof 
nets (Chaudhuri, Miller, and Saurin 2008) and classical expansion proofs (Chaudhuri, Hetzl, and Miller 2012).

Saturating focused proofs are almost maximal muli-focused proofs according to the definition of Chaudhuri, Miller, and Saurin (2008). The difference is that multi-focusing allow to focus on both variables in the context and the goal in the same time, while our right-focusing rule SAT-INTRO can only be applied sequentially after SAT (which does multi-left-focusing). To recover the exact structure of maximal multi-focusing, one would need to allow SAT to also focus on the right, and use it only when the right choices do not depend on the outcome on saturation of the left (the foci of the same set must be independent), that is when none of the bound variables are used (typically to saturate further) before the start of the next invertible phase. This is a rather artificial restriction from a backward-search perspective. Maximal multi-focusing is more elegant, declarative in this respect, but is less suited to proof search.

Equivalence of terms in presence of sums Ghani (1995) first proved the decidability of equivalence of lambda-terms with sums, using sophisticated rewriting techniques. The two works that followed (Altenkirch, Dybjer, Hofmann, and Scott 2001; Balat, Di Cosmo, and Fiore 2004) used normalization-by-evaluation instead. Finally, Lindley (2007) was inspired by Balat, Di Cosmo, and Fiore (2004) to re-explain equivalence through rewriting. Our idea of "cutting sums as early as possible" was inspired from Lindley (2007), but in retrospect it could be seen in the "restriction (A)" in the normal forms of Balat, Di Cosmo, and Fiore (2004), or directly in the "maximal conversions" of Ghani (1995).

Note that the existence of unknown atoms is an important aspect of our calculus. Without them (starting only from base types 0 and 1 ), all types would be finitely inhabited. This observation is the basis of the promising unpublished work of Ahmad, Licata, and Harper (2010), also strongly relying on (higher-order) focusing. Finiteness hypotheses also play an important role in Ilik (2014), where they are used to reason on type isomorphisms in presence of sums. Our own work does not handle 1 or 0 ; the latter at least is a notorious source of difficulties for equivalence, but is also seldom necessary in practical programming applications.

Elaboration of implicits Probably the most visible and the most elegant uses of typed-directed code inference for functional languages are type-classes (Wadler and Blott 1989) and implicits (Oliveira, Moors, and Odersky 2010). Type classes elaboration is traditionally presented as a satisfiability problem (or constraint solving problem (Stuckey and Sulzmann 2002)) that happens to have operational consequences. Implicits recast the feature as elaboration of a programming term, which is closer to our methodology. Type-classes traditionally try (to various degrees of success) to ensure coherence, namely that a given elaboration goal always give the same dynamic semantics wherever it happens in the program - often by making instance declarations a toplevel-only construct. Implicits allow a more modular construction of the elaboration environment, but have to resort to priorities to preserve determinism (Oliveira, Schrijvers, Choi, Lee, Yi, and Wadler 2014).

We propose to reformulate the question of determinism or ambiguity by presenting elaboration as a typing problem, and proving that the elaborated problems intrinsically have unique inhabitants. This point of view does not by itself solve the difficult questions of which are the good policies to avoid ambiguity, but it provides a more declarative setting to expose a given strategy; for example, priority to the more recently introduced implicit would translate to an explicit weakening construct, removing older candidates at introduction time, or a restricted variable lookup semantics.

(The global coherence issue is elegantly solved, independently of our work, by using a dependent type system where the values that semantically depend on specific elaboration choices (eg., a balanced tree ordered with respect to some specific order) have a type that syntactically depends on the elaboration witness. This approach meshes very well with our view, especially in systems with explicit equality proofs between terms, where features that grow the implicit environment could require proofs from the user that unicity is preserved.)

Smart completion and program synthesis Type-directed program synthesis has seen sophisticated work in the recent years, notably Perelman, Gulwani, Ball, and Grossman (2012), Gvero, Kuncak, Kuraj, and Piskac (2013). Type information is used to fill missing holes in partial expressions given by the users, typically among the many choices proposed by a large software library. Many potential completions are proposed interactively to the user and ordered by various ranking heuristics.

Our uniqueness criterion is much more rigid: restrictive (it has far less potential applications) and principled (there are no heuristics or subjective preferences at play). Complementary, it aims for application in richer type systems, and in programming constructs (implicits, etc.) rather than tooling with interactive feedback.

Synthesis of glue code interfacing whole modules has been presented as a type-directed search, using type isomorphisms (Aponte and Cosmo 1996) or inhabitation search in combinatory logics with intersection types (Düdder et al. 2014).

We were very interested in the recent Osera and Zdancewic (2015), which generates code from both expected type and input/output examples. The works are complementary: they have interesting proposals for data-structures and algorithm to make term search efficient, while we bring a deeper connection to prooftheoretic methods. They independently discovered the idea that saturation must use the "new" context, in their work it plays the role of an algorithmic improvement they call "relevant term generation".

\subsection{Future work}

We hope to be able to extend the uniqueness algorithm to more powerful type systems, such as System F polymorphism or dependent types. Decidability, of course, is not to be expected: deciding uniqueness is at least as hard as deciding inhabitation, and this quickly becomes undecidable for more powerful systems. Yet, we hope that the current saturation approach can be extended to give an effective semi-decision procedures. We will detail below two extensions that we have started looking at, unit and empty types, and parametric polymorphism; and two extensions we have not considered yet, substructural logics and equational reasoning.

Unit and empty types As an experiment, we have added a nonformalized support for the unit type 1 and the empty type 0 to our implementation. The unit types poses no difficulties, but we were more surprised to notice that they empty type seems also simple to handle - although we have not proved anything about it for now. We add it as a positive, with the following left-introduction rule (and no right-introduction rule):

$$
\text { SINV-EMPTY }
$$

$$
\overline{\Gamma ; \Delta, x: 0 \vdash_{\text {sinv }} \text { absurd }(x): A}
$$

Our saturation algorithm then naturally gives the expected equivalence rule in presence of 0 , which is that all programs in a inconsistent context ( 0 is provable) are equal $\left(A^{0}=1\right)$ : saturation will try to "cut all 0", and thus detect any inconsistency; if one or several proofs of 0 are found, the following invertible phase will always use the SINV-EMPTY rule, and find absurd(_) as the unique derivation. For example, while the bind function for the $A$-translation monad $B \mapsto(B \rightarrow A) \rightarrow A$ is not unique for arbitrary formulas $A$, our extended prototype finds a unique bind for the non-delimited continuation monad $B \mapsto B \rightarrow 0 \rightarrow 0$. 
Polymorphism Naively adding parametric polymorphism to the system would suggest the following rules:

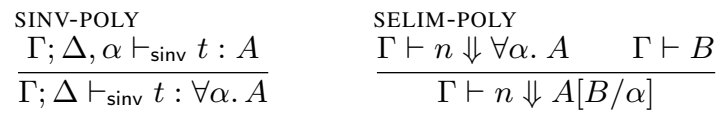

The invertible introduction rule is trivially added to our algorithm. It generalizes our treatment of atomic types by supporting a bit more than purely prenex polymorphism, as it supports all quantifiers in so-called "positive positions" (to the left of an even number of arrows), such as $1 \rightarrow(\forall \alpha . \alpha \rightarrow \alpha)$ or $((\forall \beta . \beta \rightarrow \beta) \rightarrow$ $X) \rightarrow X$. However, saturating the elimination rule SELIM-POLY would a priori require instantiating the polymorphic type with infinitely many instances (there is no clear subformula property anymore). Even naive (and probably incomplete) strategies such as instantiating with all closed formulas of the context lead to nontermination, as for example instantiating the variable $\alpha$ of closed type $1 \rightarrow \forall \alpha . \alpha$ with the closed type itself leads to an infinite regress of deduced types of the form $1 \rightarrow 1 \rightarrow 1 \rightarrow \ldots$.

Another approach would be to provide a left-introduction rule for polymorphism, based on the idea, loosely inspired by higherorder focusing (Zeilberger 2008), that destructing a value is inspecting all possible ways to construct it. For example, performing proof search determines that any possible closed proof of the term $\forall \alpha .(X \rightarrow Y \rightarrow \alpha)$ must have two subgoals, one of type $X$ and another of type $Y$; and that there are two ways to build a closed proof of $\forall \alpha .(X \rightarrow \alpha) \rightarrow(Y \rightarrow \alpha)$, using either a subgoal of type $X$ or of type $Y$. How far into the traditional territory of parametricity can we go using canonical syntactic proof search only?

Substructural logics Instead of moving to more polymorphic type systems, one could move to substructural logics. We could expect to refine a type annotation using, for example, linear arrows, to get a unique inhabitant. We observed, however, that linearity is often disappointing in getting "unique enough" types. Take the polymorphic type of mapping on lists, for example: $\forall \alpha \beta$. $(\alpha \rightarrow$ $\beta) \rightarrow($ List $\alpha \rightarrow$ List $\beta$ ). Its inhabitants are the expected map composed with any function that can reorder, duplicate or drop elements from a list. Changing the two inner arrows to be linear gives us the set of functions that may only reorder the mapped elements: still not unique. An idea to get a unique type is to request a mapping from $(\alpha \leq \beta)$ to (List $\alpha \leq$ List $\beta$ ), where the subtyping relation $(\leq)$ is seen as a substructural arrow type.

(Dependent types also allow to capture List .map, as the unique inhabitant of the dependent induction principle on lists is unique.)

Equational reasoning We have only considered pure, strongly terminating programs so far. One could hope to find monadic types that uniquely defined transformations of impure programs (e.g. $(\alpha \rightarrow \beta) \rightarrow \mathrm{M} \alpha \rightarrow \mathrm{M} \beta)$. Unfortunately, this approach would not work by simply adding the unit and bind of the monad as formal parameters to the context, because many programs that are only equal up to the monadic laws would be returned by the system. It could be interesting to enrich the search process to also normalize by the monadic laws. In the more general case, can the search process be extended to additional rewrite systems?

\section{Conclusion}

We have presented an algorithm that decides whether a given type of the simply-typed lambda-calculus with sums has a unique inhabitant modulo $\beta \eta$-equivalence; starting from standard focused proof search, the new ingredient is saturation which egarly cuts any positive that can be derived from the current context by a focused elimination. Termination is obtained through a context approximation result, remembering one or "two-or-more" variables of each type.
This is a foundational approach to questions of code inference, yet preliminary studies suggest that there are already a few potential applications, to be improved with future support for richer systems.

Of course, guessing a program from its type is not necessarily beneficial if the type is as long to write (or harder to read) than the program itself. We see code and type inference as mutuallybeneficial features, allowing the programmer to express intent in part through the term language, in part through the type language, playing on which has developped the more expressive definitions or abstractions for the task at hand.

Acknowledgments We are grateful to Adrien Guatto and anonymous reviewers for their helpful feedback. 


\section{References}

Arbob Ahmad, Daniel R. Licata, and Robert Harper. Deciding coproduct equality with focusing. Online draft, 2010.

Thorsten Altenkirch, Peter Dybjer, Martin Hofmann, and Philip J. Scott. Normalization by evaluation for typed lambda calculus with coproducts. In LICS, 2001.

Jean-Marc Andreoli. Logic Programming with Focusing Proof in Linear Logic. Journal of Logic and Computation, 1992.

Takahito Aoto. Uniqueness of normal proofs in implicational intuitionistic logic. Journal of Logic, Language and Information, 1999.

Takahito Aoto and Hiroakira Ono. Non-Uniqueness of Normal Proofs for Minimal Formulas in Implication-Conjunction Fragment of BCK. Bulletin of the Section of Logic, 1994.

Maria-Virginia Aponte and Roberto Di Cosmo. Type isomorphisms for module signatures. In PLILP, 1996.

Ali Babaev and Sergei Solov'ev. A coherence theorem for canonical morphisms in cartesian closed categories. Journal of Soviet Mathematics, 1982.

Vincent Balat, Roberto Di Cosmo, and Marcelo Fiore. Extensional normalisation and type-directed partial evaluation for typed lambda calculus with sums. In POPL, 2004.

Pierre Bourreau and Sylvain Salvati. Game semantics and uniqueness of type inhabitance in the simply-typed $\lambda$-calculus. In TLCA, 2011.

Taus Brock-Nannestad and Carsten Schürmann. Focused natural deduction. In $L P A R-17,2010$.

Sabine Broda and Luís Damas. On long normal inhabitants of a type. $J$. Log. Comput., 2005.

Kaustuv Chaudhuri and Frank Pfenning. Focusing the inverse method for linear logic. In CSL, 2005.

Kaustuv Chaudhuri, Dale Miller, and Alexis Saurin. Canonical sequent proofs via multi-focusing. In IFIP TCS, 2008.

Kaustuv Chaudhuri, Stefan Hetzl, and Dale Miller. A Systematic Approach to Canonicity in the Classical Sequent Calculus. In CSL, 2012.

Pierre-Louis Curien and Guillaume Munch-Maccagnoni. The duality of computation under focus. In IFIP TCS, 2010.

Anatoli Degtyarev and Andrei Voronkov. Introduction to the inverse method. In Handbook of Automated Reasoning. 2001.

Kosta Dosen. Identity of proofs based on normalization and generality. Bulletin of Symbolic Logic, 2003.

Gilles Dowek and Ying Jiang. On the expressive power of schemes. Inf. Comput., 2011.

Boris Düdder, Moritz Martens, and Jakob Rehof. Staged composition synthesis. In ESOP, 2014.

Roy Dyckhoff. Contraction-free sequent calculi for intuitionistic logic. J. Symb. Log., 1992

Roy Dyckhoff. Intuitionistic decision procedures since gentzen, 2013. Talk notes.

Didier Galmiche and Daniel Méry. A connection-based characterization of bi-intuitionistic validity. J. Autom. Reasoning, 2013.

Neil Ghani. Beta-eta equality for coproducts. In TLCA, 1995.

Tihomir Gvero, Viktor Kuncak, Ivan Kuraj, and Ruzica Piskac. Complete completion using types and weights. In PLDI, 2013.

Hugo Herbelin. A lambda-calculus structure isomorphic to gentzen-style sequent calculus structure. In CSL, 1993.

Danko Ilik. Axioms and decidability for type isomorphism in the presence of sums. CoRR, 2014. URL http: //arxiv .org/abs/1401.2567.

Edward Kmett. Lens, 2012. URL https://github.com/ekmett/lens.

Edward Kmett. Lens wiki - types, 2013. URL https://github.com/ ekmett/lens/wiki/Types.

Neelakantan R. Krishnaswami. Focusing on pattern matching. In POPL, 2009.

Olivier Laurent. A proof of the focalization property of linear logic. 2004.
Chuck Liang and Dale Miller. Focusing and polarization in intuitionistic logic. CoRR, 2007. URL http: //arxiv . org/abs/0708.2252.

Sam Lindley. Extensional rewriting with sums. In TLCA, 2007.

Pablo López, Frank Pfenning, Jeff Polakow, and Kevin Watkins. Monadic concurrent linear logic programming. In PPDP, 2005.

Sean McLaughlin and Frank Pfenning. Imogen: Focusing the polarized inverse method for intuitionistic propositional logic. In $L P A R, 2008$.

Dale Miller and Alexis Saurin. From proofs to focused proofs: A modular proof of focalization in linear logic. In CSL, 2007.

Grigori Mints. Closed categories and the theory of proofs. Journal of Soviet Mathematics, 1981.

Bruno C. d. S. Oliveira, Adriaan Moors, and Martin Odersky. Type classes as objects and implicits. In OOPSLA, 2010.

Bruno C. d. S. Oliveira, Tom Schrijvers, Wontae Choi, Wonchan Lee, Kwangkeun Yi, and Philip Wadler. The implicit calculus: A new foundation for generic programming. 2014.

Peter-Michael Osera and Steve Zdancewic. Type-and-example-directed program synthesis. In $P L D I, 2015$.

Jens Otten and Christoph Kreitz. A uniform proof procedure for classical and non-classical logics. In KI Advances in Artificial Intelligence, 1996.

Daniel Perelman, Sumit Gulwani, Thomas Ball, and Dan Grossman. Typedirected completion of partial expressions. In PLDI, 2012.

Gabriel Scherer. Mining opportunities for unique inhabitants in dependent programs, 2013.

Gabriel Scherer. 2-or-more approximation for intuitionistic logic. 2014.

Gabriel Scherer and Didier Rémy, 2015. URL http://gallium.inria. $\mathrm{fr} / \sim$ scherer/research/unique_inhabitants/.

Robert J. Simmons. Structural focalization. 2011. URL http://arxiv . org/abs/1109.6273.

Colin Stirling. Proof systems for retracts in simply typed lambda calculus. In Automata, Languages, and Programming - ICALP, 2013.

Peter J. Stuckey and Martin Sulzmann. A theory of overloading. In ICFP, 2002.

Nikolay Vorob'ev. A new algorithm of derivability in a constructive calculus of statements. In Problems of the constructive direction in mathematics, 1958.

Philip Wadler and Stephen Blott. How to make ad-hoc polymorphism less ad-hoc. In POPL, 1989.

Lincoln A. Wallen. Automated proof search in non-classical logics: Efficient matrix proof methods for modal and intuitionistic logic, 1987.

Joe B. Wells and Boris Yakobowski. Graph-based proof counting and enumeration with applications for program fragment synthesis. In LOPSTR, 2004.

Marek Zaoinc. Fixpoint technique for counting terms in typed lambdacalculus. Technical report, State University of New York, 1995.

Noam Zeilberger. Focusing and higher-order abstract syntax. In POPL, 2008. 


\section{A. The saturation algorithm as a system of inference rules}

In Figure 9 we present a complete set of inference rules that captures the behavior of our search algorithm.

Data structures The judgments uses several kinds data-structures.

- 2-sets $S, T \ldots$, are sets restricted to having at most two (distinct) elements; we use $\{\ldots\}_{2}$ to build a 2 -set, and $\left(\cup_{2}\right)$ for union of two-sets (keeping at most two elements in the resulting union). We use the usual notation $x \in S$ for 2-set membership. To emphasize the distinction, we will sometimes write $\{\ldots\}_{\infty}$ for the usual, unbounded sets. Remark that 2 -sets correspond to the "plurality monad" of Section 5: a monad is more convenient to use in an implementation, but for inference rules we use the set-comprehension notation.

- 2-mappings are mappings from a set of keys to 2-sets. In particular, $\Gamma$ denotes a 2 -mapping from negative or atomic types to 2 -sets of formal variables. We use the application syntax $\Gamma(A)$ for accessing the 2 -set bound to a specific key, $A \mapsto S$ for the singleton mapping from one variable to one 2-set, and $(\oplus)$ for the union of 2-mappings, which applies $\left(\cup_{2}\right)$ pointwise:

$$
\left(\Gamma \oplus \Gamma^{\prime}\right)(A) \stackrel{\text { def }}{=} \Gamma(A) \cup_{2} \Gamma^{\prime}(A)
$$

Finally, we write $\emptyset$ for the mapping that maps any key to the empty 2-set.

- multisets $M$ are mappings from elements to a natural number count. The "memories" of subgoal ancestors are such mappings (where the keys are "judgments" of the form $\Gamma \vdash A$ ), and our rules will guarantee that the value of any key is at most 2 . We use the application syntax $M(\Gamma \vdash A)$ to access the count of any element, and $(+)$ for pointwise addition of multisets:

$$
\left(M+M^{\prime}\right)(\Gamma \vdash A) \stackrel{\text { def }}{=} M(\Gamma \vdash A)+M^{\prime}(\Gamma \vdash A)
$$

- (ordered) lists $\Sigma$

Finally, we use a substraction operation $(-2)$ between 2mappings, that can be defined from the 2-set restriction operation $S \backslash_{2} n$ (where $n$ is a natural number in $\{0,1,2\}$ ). Recall that $\# S$ is the cardinal of the set (or 2-set) $S$.

$$
\begin{gathered}
\left(\Gamma^{\prime}-{ }_{2} \Gamma\right)(A) \stackrel{\text { def }}{=} \Gamma^{\prime}(A) \backslash_{2} \#(\Gamma(A)) \\
S \backslash_{2} 0 \stackrel{\text { def }}{=} S \\
\emptyset \backslash_{2} 1 \stackrel{\text { def }}{=} \emptyset \quad\{a, \ldots\}_{2} \backslash_{2} 1 \stackrel{\text { def }}{=}\{a\}_{2} \\
S \backslash_{2} 2 \stackrel{\text { def }}{=} \emptyset
\end{gathered}
$$

Note that $\{a, b\} \backslash_{2} 1$ is not uniquely defined: it could be either $a$ or $b$, the choice does not matter. The defining property of $S \backslash_{2} n$ is that it is a minimal 2-set $S^{\prime}$ such as $S^{\prime} \cup_{2} T=S$.

Judgments The algorithm is presented as a system of judgmentdirected (that is, directed by the types in the goal and the context(s)) inference rules. It uses the following five judgment forms:

- invertible judgments $M @ \Gamma ; \Gamma^{\prime} ; \Sigma \vdash_{\text {inv }}^{\text {alg }} S: A$

- saturation judgments $M @ \Gamma ; \Gamma^{\prime} \vdash_{\text {sat }}^{\text {alg }} S: P_{\text {at }}$

- post-saturation judgments $M @ \Gamma \vdash_{\text {post }}^{\mathrm{alg}} S: A$

- introduction judgments $M @ \Gamma \vdash^{\text {alg }} S \Uparrow A$

$$
\begin{aligned}
& \text { ALG-SINV-SUM } \\
& M @ \Gamma ; \Gamma^{\prime} ; x: A, \Sigma \vdash_{\text {inv }}^{\text {alg }} S: C \\
& M @ \Gamma ; \Gamma^{\prime} ; x: B, \Sigma \vdash_{\text {inv }}^{\text {alg }} T: C \\
& \hline M @ \Gamma ; \Gamma^{\prime} ; x: A+B, \Sigma \vdash_{\text {inv }}^{\text {alg }} \delta(x, x . S, x . T): C
\end{aligned}
$$

ALG-SINV-PROD

$\frac{M @ \Gamma ; \Gamma^{\prime} ; \emptyset \vdash_{\text {inv }}^{\text {alg }} S: A \quad M @ \Gamma ; \Gamma^{\prime} ; \emptyset \vdash_{\text {inv }}^{\text {alg }} T: B}{M @ \Gamma ; \Gamma^{\prime} ; \emptyset \vdash_{\text {inv }}^{\text {alg }}(S, T): A * B}$

ALG-SINV-ARR

$\frac{M @ \Gamma ; \Gamma^{\prime} ; x: A \vdash_{\text {inv }}^{\mathrm{alg}} S: B}{M @ \Gamma ; \Gamma^{\prime} ; \emptyset \vdash_{\mathrm{inv}}^{\mathrm{alg}} \lambda x . S: A \rightarrow B}$

ALG-SINV-RELEASE

$\frac{M @ \Gamma ; \Gamma^{\prime} \oplus\left(N_{\mathrm{at}} \mapsto\{x\}_{2}\right) ; \Sigma \vdash_{\mathrm{inv}}^{\mathrm{alg}} S: A}{M @ \Gamma ; \Gamma^{\prime} ; x: N_{\mathrm{at}}, \Sigma \vdash_{\mathrm{inv}}^{\mathrm{alg}} S: A}$

$$
\begin{aligned}
& \text { ALG-SINV-END ALG-SAT-KILL } \\
& \frac{M @ \Gamma ;\left(\Gamma^{\prime}-{ }_{2} \Gamma\right) \vdash_{\mathrm{sat}}^{\mathrm{alg}} S: P_{\mathrm{at}}}{M @ \Gamma ; \Gamma^{\prime} ; \emptyset \vdash_{\mathrm{inv}}^{\mathrm{alg}} S: P_{\mathrm{at}}} \quad \frac{M\left(\Gamma \vdash P_{\mathrm{at}}\right)=2}{M @ \Gamma ; \emptyset \vdash_{\mathrm{sat}}^{\mathrm{alg}} \emptyset: P_{\mathrm{at}}} \\
& \text { ALG-SAT-POST } \\
& \frac{M\left(\Gamma \vdash P_{\mathrm{at}}\right)<2 \quad M \oplus_{2}(\Gamma \vdash P) @ \Gamma \vdash_{\mathrm{post}}^{\mathrm{alg}} S: P_{\mathrm{at}}}{M @ \Gamma ; \emptyset \vdash_{\text {sat }}^{\mathrm{alg}} S: P_{\mathrm{at}}} \\
& \text { ALG-POST-INTRO ALG-POST-ATOM } \\
& \frac{M @ \Gamma \vdash^{\text {alg }} S \Uparrow P}{M @ \Gamma \vdash_{\text {post }}^{\text {alg }} S: P} \quad \frac{M @ \Gamma \vdash^{\text {alg }} S \Downarrow X}{M @ \Gamma \vdash_{\text {post }}^{\text {alg }} S: X}
\end{aligned}
$$

ALG-SAT

$$
\begin{gathered}
\forall\left(P \mid P \text { subformula }\left(\Gamma, \Gamma^{\prime}\right)\right), \quad \Gamma_{P} \stackrel{\text { def }}{=} \bigcup_{2}\left\{S_{\text {ne }} \mid M @ \Gamma, \Gamma^{\prime} \vdash^{\text {alg }} S_{\text {ne }} \Downarrow P\right\} \\
B \stackrel{\text { def }}{=} \bigoplus_{P}\left\{P \mapsto\left\{x_{n}\right\}_{2} \mid n \in S_{P}\right\} \\
M \stackrel{@}{ } \Gamma, \Gamma^{\prime} ; \emptyset ; B \vdash_{\text {inv }}^{\text {alg }} S: Q_{\text {at }} \\
S^{\prime} \stackrel{\text { def }}{=} \begin{cases}\text { let } \left.\bar{x}=\bar{n} \text { in } t \mid \begin{array}{l}
t \in S, \\
(\bar{x}, \bar{n}) \stackrel{\text { def }}{=}\left\{\left(x_{n}, n\right) \mid x_{n} \in B(P), t \text { uses } x_{n}\right\}_{\infty}
\end{array}\right\}_{2} \\
M @ \Gamma ; \Gamma^{\prime} \vdash_{\text {sat }}^{\text {alg }} S^{\prime}: Q_{\text {at }}\end{cases}
\end{gathered}
$$$$
\begin{aligned}
& \text { ALG-SINTRO-SUm } \\
& M @ \Gamma \vdash^{\text {alg }} S \Uparrow A \quad M @ \Gamma \vdash^{\text {alg }} T \Uparrow B \\
& M @ \vdash^{\text {alg }}\left(\sigma_{1} S\right) \cup_{2}\left(\sigma_{2} T\right) \Uparrow A+B
\end{aligned}
$$$$
\text { ALG-SINTRO-END }
$$$$
\frac{M @ \Gamma ; \emptyset ; \emptyset \vdash_{\text {inv }}^{\text {alg }} S: N_{\text {at }}}{M @ \Gamma \vdash^{\text {alg }} S \Uparrow N_{\text {at }}}
$$

$$
\begin{gathered}
A \text { subformula } \Gamma \\
S_{\text {var }} \stackrel{\text { def }}{=} \Gamma(A) \\
\frac{S_{\text {proj }} \stackrel{\text { def }}{=} \bigcup_{2}\left\{\pi_{i} S \mid M \stackrel{\text { app }}{\stackrel{\text { def }}{=}} \bigcup_{2}\left\{S T \mid M \vdash^{\text {alg }} S \Downarrow B_{1} * B_{2}, B_{i}=A\right\}\right.}{\left.M \vdash^{\text {alg }} S \Downarrow B \rightarrow A, M @ \Gamma \vdash^{\text {alg }} T \Uparrow B\right\}} \\
M \text { @ }^{\text {alg }} S_{\text {var }} \cup_{2} S_{\text {proj }} \cup_{2} S_{\text {app }} \Downarrow A
\end{gathered}
$$

Figure 9. Saturation algorithm 
- elimination judgments $M @ \Gamma \vdash^{\text {alg }} S \Downarrow A$

All algorithmic jugments respect the same conventions:

- $M$ is a memory (remembering ancestors judgments for termination), a multiset of judgments of the form $\Gamma \vdash A$

- $\Gamma, \Gamma^{\prime}$ are 2-mappings from negative or atomic types to 2-sets of formal variables (we will call those "contexts")

- $\Sigma$ is an ordered list of pairs $x: A$ of formal variables and unrestricted types

- $S$ is a 2-set of proof terms of the saturating focused logic

- $A$ is a type/formula

The $S$ position is the output position of each judgment (the algorithm returns a 2-set of distinct proof terms); all other positions are input positions; any judgment has exactly one applicable rule, determined by the value of its input positions.

Sets of terms We extend the term construction operations to 2sets of terms:

$\begin{array}{lll}\lambda x . S & \stackrel{\text { def }}{=} & \{\lambda x . t \mid t \in S\}_{2} \\ S T & \stackrel{\text { def }}{=} & \{t u \mid t \in S, u \in T\}_{2} \\ (S, T) & \stackrel{\text { def }}{=} & \{(t, u) \mid t \in S, u i n T\}_{2} \\ \pi_{i} S & \stackrel{\text { def }}{=} & \left\{\pi_{i} t \mid t \in S\right\}_{2} \\ \sigma_{i} S & \stackrel{\text { def }}{=} & \left\{\sigma_{i} t \mid t \in S\right\}_{2} \\ \delta(x, x . S, x . T) & \stackrel{\text { def }}{=} & \{\delta(x, x . t, x . u) \mid t \in S, u \in T\}_{2}\end{array}$

Invertible rules The invertible focused rules $\Gamma ; \Delta \vdash_{\text {inv }} ?: A$ exhibit "don't care" non-determinism in the sense that their order of application is irrelevant and captured by invertible commuting conversions (see Section 2.1). In the algorithmic judgment, we enforce a specific order through the two following restrictions.

The unrestricted context $\Delta$ is split into both a negative context (as a 2-mapping) $\Gamma^{\prime}$ and a not-processed-yet unrestricted context $\Sigma$. By using an ordered list for the unrestricted context, we fix the order in which positives in the context are deconstructed. When the head of the ordered list has been fully deconstructed (it is negative or atomic), the new rule ALG-SINV-RELEASE moves it into the known-negative context $\Gamma^{\prime}$.

The invertible right-introduction rules are restricted to judgments whose ordered context $\Sigma$ is empty. This enforces that leftintroductions are always applied fully before any right-introduction. Note that we could arbitrarily decide to enforce the opposite order by un-restricting right-introduction rules, and requiring that left-introduction (and releases) only happen when the succedent is positive or atomic.

Finally, the final invertible rule ALG-SINV-END uses 2-mapping substractions $\Gamma-{ }_{2} \Gamma^{\prime}$ to trim the new context $\Gamma^{\prime}$ before handing it to the saturation rules: for any given formula $N_{\text {at }}$, all bindings for $N_{\text {at }}$ are removed from $\Gamma^{\prime}$ if there are already two in $\Gamma$, and at most one binding is kept if there is already one in $\Gamma$. Morally, the reason why it is correct to trim (that is, it does not endanger unicity completeness $(\S 1.5)$ is that the next rules in bottom-up search will only use the merged context $\Gamma \cup_{2} \Gamma^{\prime}$ (which is preserved by trimming by construction of $(-2)$ ), or saturate with bindings from $\Gamma^{\prime}$. Any strictly positive that can be deduced by using one of the variables present in $\Gamma^{\prime}$ but removed from $\Gamma \cup_{2} \Gamma^{\prime}$ has already been deduced from $\Gamma$. It is useful to trim in this rule (we could trim much more often) because subsequent saturated rules will test the new context $\Gamma^{\prime}-{ }_{2} \Gamma$ for emptyness, so it is interesting to minimize it. In any case, we need to trim in at least one place in order for typing judgments not to grow unboundedly.

Saturation rules If the (trimmed) new context is empty, we test whether the judgment of the current subgoal has already occurred twice among its ancestors; in this case, the rule ALG-SAT-KILL terminates the search process by returning the empty 2 -set of proof terms. In the other case, the number of occurences of this judgment is incremented in the rule ALG-SAT-POST, and one of the (transparent) "post-saturation" rules ALG-POST-INTRO or ALG-POST-ATOM are applied.

This is the only place where the memory $M$ is accessed and updated. The reason why this suffices is any given phase (invertible phase, or phase of non-invertible eliminations and introductions) is only of finite length, and either terminates or is followed by a saturation phase; because contexts grow monotonously in a finite space (of 2-mappings rather than arbitrary contexts), the trimming of rule ALG-SINV-END returns the empty context after a finite number of steps: an infinite search path would need to go through ALG-SAT-POST infinitely many times, and this suffices to prove termination.

The most important and complex rule is ALG-SAT, which proceeds in four steps. First, we compute the 2 -set $S_{P}$ of all ways to deduce any strict positive $P$ from the context - for any $P$ we need not remember more than two ways. We know that we need only look for $P$ that are deducible by elimination from the context $\Gamma, \Gamma^{\prime}$ - the finite set of subformulas is a good enough approximation. Second, we build a context $B$ binding a new formal variable $x_{n}$ for each elimination neutral $n-$ it is crucial for canonicity that all $n$ are new and semantically distinct from each other at this point, otherwise duplicate bindings would be introduced. Third, we compute the set $S$ of all possible (invertible) proofs of the goal under this saturation context $B$. Fourth, we filter the elements of $S$ to retain, for each of them, only the bindings that were actually used this requires an application of weakening as a meta-property, as the proof terms are supposed to live under the context extended with the full binding $B$.

Non-invertible introduction and elimination rules The introduction rule ALG-SINTRO-SUM collects solutions using either left or right introductions, and unites them in the result 2-set. Similarly, all elimination rules are merged in one single rule, which corresponds to all ways to deduce a given formula $A$ : directly from the context (if it is negative or atomic), by projection of a pair, or application of a function. The search space for this sequent is finite, as goal types grow strictly at each type, and we can kill search for any type that does not appear as a subformula of the context.

(The inference-rule presentation differs from our OCaml implementation at this point. The implementation is more effective, it uses continuation-passing style to attempt to provide function arguments only for the applications we know are found in context and may lead to the desired result. Such higher-order structure is hard to render in an inference rule, so we approximated it with a more declarative presentation here. This is the only such simplification.)

The properties of this algorithm are described and proved in Section B.4, to justify the correctness Theorem 4 of Section 5.

\section{B. Proofs}

\section{B.1 Focusing}

Fact (1). The focused intuitionistic logic is complete with respect to intuitionistic logic. It is also computationally complete: any welltyped lambda-term is $\beta \eta$-equivalent to (the let-substitution of) a proof witness of the focused logic.

Logical completeness. This system naturally embeds into the system LJF of Liang and Miller (2007) (by polarizing the products and all atoms negatively), which is proved sound, and complete for any polarization choice. 
Computational completeness could be argued to be folklore, or a direct adaptation of previous work on completeness of focusing: a careful lecture of the elegant presentation of Simmons (2011) (or Laurent (2004) for linear logic) would show that its logical completeness argument in fact proves computational correctness. Without sums, it exactly corresponds to the fact that $\beta$-short $\eta$-long normal forms are computable for well-typed lambda-terms of the simply-typed calculus.

However, our use of let-bindings in the term calculus makes our system slightly more exotic. We will thus introduce an explicit $\eta$-expanding, let-introducing transformation from $\beta$-normal forms to valid focused proofs for our system. Detailing this transformation also serves by building intuition for the computational completeness proof of the saturating focused logic.

Computational completeness. Let us remark that simply-typed lambda-calculus without fixpoints is strongly terminating, and write $\mathrm{NF}_{\beta}(t)$ for the (full) $\beta$-normal form of $t$.

We define in Figure 10 an expansion relation $\Gamma ; \Delta \vdash t \rightsquigarrow t^{\prime}: A$ that turns any well-typed $\beta$-normal form $\Gamma, \Delta \vdash t: A$ into a valid focused derivation $\Gamma ; \Delta \vdash_{\text {inv }} t^{\prime}: A$.

We use three mutually recursive judgments, the invertible and focusing translations $\Gamma ; \Delta \vdash t \rightsquigarrow t^{\prime}: A$ and $\Gamma \vdash_{\text {foc }} t \rightsquigarrow t^{\prime}: P_{\text {at }}$, and the neutral translation $\Gamma \vdash_{\text {ne }} n \rightsquigarrow n^{\prime} \Downarrow A$. For the two first judgments, the inputs are the context(s), source term, and translation type, and the output is the translated term. For the neutral judgment the translation type is an output - this reversal follows the usual bidirectional typing of normal forms.

Two distinct aspects of the translation need to be discussed:

1. Finiteness. It is not obvious that a translation derivation $\Gamma ; \Delta \vdash$ $t \rightsquigarrow t^{\prime}: A$ exists for any $\Gamma, \Delta \vdash t: A$, because subderivations of invertible rules perform $\beta$-normalization of their source term, which may a priori make it grow without bounds. It could be the case that for certain source terms, there does not exist any finite derivation.

2. Partiality. As the rules are neither type- nor syntax-directed, it is not obvious that any input term, for example $\delta\left(t_{1} t_{2}, x_{1} . u_{1}, x_{2} . u_{2}\right)$, has a matching translation rule.

3. Non-determinism. The invertible rules are not quite typeddirected, and the REW-FOC-ELIM rule is deeply non-determinist, as it applies for any neutral subterm of the term being translated - that is valid in the current typing environment. This non-determinism allows the translation to accept any valid focused derivation for an input term, reflecting the large choice space of when to apply the FOC-ELIM rule in backward focused proof search.

Totality The use of $\beta$-normalization inside subderivations precisely corresponds to the "unfocused admissibility rules" of Simmons (2011). To control the growth of subterms in the premises of rules, we will use as a measure (or accessibility relation) the two following structures, from the less to the more important in lexicographic order:

- The (measure of the) types in the context(s) of the rewriting relation. This measure is strictly decreasing in the invertible elimination rule for sums, but increasing for the arrow introduction rule.

- The (measure of the) type of the goal of the rewriting relation. This measure is strictly decreasing in the introduction rules for arrow, products and sums, but increasing in REW-FOC-ELIM or neutral rules.

- The set of (measures of) translation judgments $\Gamma \vdash_{\text {ne }} n \rightsquigarrow n^{\prime} \Downarrow A$ for well-typed neutral subterms $n$ of the translated term whose type $A$ is of maximal mesaure.
$\begin{array}{ll}\Gamma::=\operatorname{varmap}\left(N_{\text {at }}\right) & \text { negative or atomic context } \\ \Delta::=\operatorname{varmap}(A) & \text { general context }\end{array}$

REW-INV-SUM

$\Gamma ; \Delta, x: A \vdash \mathrm{NF}_{\beta}\left(t\left[\sigma_{1} x / x\right]\right) \rightsquigarrow t_{1}^{\prime}: C$

$\Gamma ; \Delta, x: B \vdash \mathrm{NF}_{\beta}\left(t\left[\sigma_{2} x / x\right]\right) \rightsquigarrow t_{2}^{\prime}: C$

$\overline{\Gamma ; \Delta, x: A+B \vdash t \rightsquigarrow \delta\left(x, x . t_{1}^{\prime}, x \cdot t_{2}^{\prime}\right): C}$

REW-INV-ARROW

$\frac{\Gamma ; \Delta, x: A \vdash \mathrm{NF}_{\beta}(t x) \rightsquigarrow u^{\prime}: B}{\Gamma ; \Delta \vdash t \rightsquigarrow \lambda x \cdot u^{\prime}: A \rightarrow B}$

REW-INV-PROD

\begin{tabular}{|c|c|}
\hline$\Gamma ; \Delta \vdash \mathrm{NF}_{\beta}\left(\pi_{1} t\right) \rightsquigarrow u_{1}^{\prime}: A_{1}$ & $\Gamma ; \Delta \vdash \mathrm{NF}_{\beta}\left(\pi_{2} t\right) \rightsquigarrow u_{2}^{\prime}: A_{2}$ \\
\hline \multicolumn{2}{|c|}{$\Gamma ; \Delta \vdash t \rightsquigarrow\left(u_{1}^{\prime}, u_{2}^{\prime}\right): A_{1} * A_{2}$} \\
\hline $\begin{array}{l}\text { REW-INV-FOC } \\
\Gamma, \Gamma^{\prime} \vdash_{\text {foc }} t \rightsquigarrow t^{\prime}: P_{\text {at }}\end{array}$ & $\begin{array}{l}\text { REW-FOC-ATOM } \\
\Gamma \vdash \vdash_{\mathrm{pe}} n \rightsquigarrow n^{\prime} \Downarrow X\end{array}$ \\
\hline$\Gamma ; \Gamma^{\prime} \vdash t \rightsquigarrow t^{\prime}: P_{\mathrm{at}}$ & $\overline{\Gamma \vdash_{\text {foc }} n \rightsquigarrow n^{\prime}: X}$ \\
\hline \multicolumn{2}{|c|}{$\begin{array}{l}\text { REW-FOC-INTRO } \\
\Gamma \vdash t \rightsquigarrow t^{\prime} \Uparrow P\end{array}$} \\
\hline$\overline{\Gamma \vdash_{f}}$ & $\rightarrow t^{\prime}: P$ \\
\hline
\end{tabular}

REW-FOC-ELIM

$$
\begin{gathered}
\Gamma, x: P \vdash C[x]: Q_{\text {at }} \\
\Gamma \vdash_{\text {ne }} n \rightsquigarrow n^{\prime} \Downarrow P \quad \Gamma ; x: P \vdash C[x] \rightsquigarrow t^{\prime}: Q_{\text {at }} \\
\hline \vdash_{\text {foc }} C[n] \rightsquigarrow \operatorname{let} x=n^{\prime} \text { in } t^{\prime}: Q_{\text {at }}
\end{gathered}
$$

$$
\begin{array}{ll}
\begin{array}{l}
\text { REW-UP-SUM } \\
\Gamma \vdash t \rightsquigarrow t^{\prime} \Uparrow A_{i}
\end{array} & \frac{\text { REW-UP-INV }}{\Gamma ; \emptyset \vdash t \rightsquigarrow t^{\prime}: N_{\mathrm{at}}} \\
\hline \vdash \sigma_{i} t \rightsquigarrow \sigma_{i} t^{\prime} \Uparrow A_{1}+A_{2} & \frac{\Gamma \mapsto t^{\prime} \Uparrow N_{\mathrm{at}}}{\Gamma \vdash t}
\end{array}
$$$$
\text { REW-UP-VAR REW-UP-PAIR }
$$$$
\frac{\left(x: N_{\mathrm{at}}\right) \in \Gamma}{\Gamma \vdash_{\mathrm{ne}} x \rightsquigarrow x \Downarrow N_{\mathrm{at}}} \quad \frac{\Gamma \vdash_{\mathrm{ne}} n \rightsquigarrow n^{\prime} \Downarrow A_{1} * A_{2}}{\Gamma \vdash_{\mathrm{ne}} \pi_{i} n \rightsquigarrow \pi_{i} n^{\prime} \Downarrow A_{i}}
$$

REW-UP-ARROW

$$
\frac{\Gamma \vdash_{\text {ne }} n \rightsquigarrow n^{\prime} \Downarrow A \rightarrow B \quad \Gamma \vdash t: A}{\Gamma \vdash_{\text {ne }} n t \rightsquigarrow n^{\prime} t^{\prime} \Downarrow B}
$$

Figure 10. Translation into focused terms

Note that while that complexity seems to increase in the premises of the judgment $\Gamma \vdash_{\text {ne }} n \rightsquigarrow n^{\prime} \Downarrow A$, this judgment should be read top-down: all the sub-neutrals of $n$ already appear as subterms of the source $t$ in the REW-FOC-ELIM application $\Gamma \vdash_{\text {foc }} t \rightsquigarrow ?: Q_{\text {at }}$ that called $\Gamma \vdash_{\text {ne }} n \rightsquigarrow ? \Downarrow A$.

This measure is non-increasing in all non-neutral rules other than REW-FOC-ELIM, in particular the rules that require renormalization ( $\beta$-reduction or $\eta$-reduction may at best duplicate the occurences of the neutral of maximal type, but not create new neutrals at higher types). In the sum-elimination rule, the neutral $x$ of type $A+B$ is shadowed by another neutral $x$ of smaller type $(A$ or $B)$. In the arrow rule, a new neutral $t x$ is introduced if $t$ is already neutral, but then $t x: B$ is at a strictly smaller type than $t: A \rightarrow B$. In the product rule, new neutral $\pi_{i} t: A_{i}$ are introduced if $t: A_{1} * A_{2}$ is neutral, but again at strictly smaller types.

Finally, this measure is strictly decreasing when applying REW-FOC-ELIM, except in the case where the chosen subterm $n \in t$ is in fact equal to $t$ where it is only non-decreasing but this can only happen once in a row, as the next call is then 
necessarily on a variable, that contains no neutral subterm of strictly positive type.

This three-fold measures proves termination of $\Gamma ; \Delta \vdash t \rightsquigarrow ?: A$ seen as an algorithm: we have proved that there are no infinite derivations for the translation judgments.

Partiality The invertible translation rules are type-directed; the neutral translation rules are directed by the syntax of the neutral source term. But the focusing translation rules are neither typenor source-directed. We have to prove that one of those three rule applies for any term - assuming that the context is negative or atomic, and the goal type positive or atomic.

The term $t$ either starts with a constructor (introduction form), a destructor (elimination form), or it is a variable; a constructor may be neither a $\lambda$ or a pair, as we assumed the type is positive or atomic. It it starts with a non-empty series of sum injections, followed by a negative or atomic term, we can use REW-FOC-INTRO. Otherwise it contains (possibly after some sum injections) a positive subterm that does not start with a constructor.

If it starts with an elimination form or a variable, it may or may not be a neutral term. If it is neutral, then one of the rules REW-FOC-ATOM (if the goal is atomic) or REW-FOC-INTRO (if the goal is strictly positive) applies. If it is not neutral (in particular not a variable), it has an elimination form applied to a subterm of the form $\delta\left(t, x_{1} . u_{1}, x_{2} . u_{2}\right)$; but then (recursively) either $t$ is a (strictly positive) neutral, or of the same form, and the rule REW-FOC-ELIM is eventually applicable.

We have proved that for any well-typed $\Gamma, \Delta \vdash t \rightsquigarrow t: A$, there exists a translation derivation $\Gamma ; \Delta \vdash t \rightsquigarrow t^{\prime}: A$ for some $t^{\prime}$.

Non-determinism The invertible rules may be applied in any order; this means that for any $t^{\prime}$ such that $\Gamma ; \Delta \vdash t \rightsquigarrow t^{\prime}: A$, for any $t^{\prime \prime}={ }_{\text {icc }} t^{\prime}$ we also have $\Gamma ; \Delta \vdash t \rightsquigarrow t^{\prime \prime}: A$ : a non-focused term translates to a full equivalence class of commutative conversions.

The rule REW-FOC-ELIM may be applied at will (as soon as the let-extruded neutral $n$ is well-typed in the current context). Applying this rule eagerly would give a valid saturated focused deduction. Not enforcing its eager application allows (but we need not formally prove it) any $\beta \eta$-equivalent focused proof to be a target of the translation.

Validity We prove by immediate (mutual) induction that, if $\Gamma, \Delta \vdash t: A$ holds, then the focusing translations are valid:

- if $\Gamma ; \Delta \vdash t \rightsquigarrow t^{\prime}: A$ then $\Gamma ; \Delta ; t^{\prime} \vdash_{\text {inv }} A$ :

- if $\Delta=\emptyset$ and $\Gamma \vdash_{\text {foc }} t \rightsquigarrow t^{\prime}: P_{\text {at }}$ then $\Gamma \vdash_{\text {foc }} t^{\prime}: P_{\text {at }}$

- if $\Delta=\emptyset$ and $\Gamma \vdash_{\text {ne }} n \rightsquigarrow n^{\prime} \Downarrow A$ then $\Gamma \vdash n^{\prime} \Downarrow A$

- if $\Delta=\emptyset$ and $\Gamma \vdash t \rightsquigarrow t^{\prime} \Uparrow A$ then $\Gamma \vdash t^{\prime} \Uparrow A$

Soundness Finally, we prove that the translation preserves $\beta \eta$ equivalence. If $\Gamma, \Delta \vdash t: A$ and $\Gamma ; \Delta \vdash t \rightsquigarrow t^{\prime}: A$, then $t={ }_{\beta \eta} t^{\prime}$ (more precisely, $t={ }_{\beta \eta}$ letexp $\left(t^{\prime}\right)$ ).

As for validity, this is proved by mutual induction on all judgments. The interesting cases are the invertible rules and the focusing elimination rule; all other cases are discarded by immediate induction.

The invertible rules correspond to an $\eta$-expansion step. For REW-INV-PROD, we have that $t={ }_{\eta}\left(\pi_{1} t, \pi_{2} t\right)$, and can thus deduce by induction hypothesis that $t={ }_{\beta \eta}\left(u_{1}^{\prime}, u_{2}^{\prime}\right)$. For REW-INV-ARROW, we have that $t={ }_{\eta} \lambda x$. $t$, and can thus deduce by induction hypothesis that $t=\beta_{\beta \eta} \lambda x . t^{\prime}$. For REW-INV-SUM, let us write $t$ as $C[x]$ with $x \notin C$, we have that

$$
\begin{array}{rlrl}
t & = & C[x: A+B] \\
& ={ }_{\eta} & \delta\left(x, x . C\left[\sigma_{1} x\right], x . C\left[\sigma_{2} x\right]\right) \\
& = & & \delta\left(x, x . t\left[\sigma_{1} x / x\right], x . t\left[\sigma_{2} x / x\right]\right) \\
& =\beta \eta & & \delta\left(x, x . t_{1}^{\prime}, x . t_{2}^{\prime}\right)
\end{array}
$$

(by induction hypothesis)

In the case of the rule REW-FOC-ELIM, the fundamental transformation is the let-binding that preserves $\beta \eta$-equivalence.

$$
\begin{aligned}
t & = & & t[x / n][n / x] \\
& =\beta \eta & & \text { let } x=n \text { in } t[x / n] \\
& =\beta \eta & & \text { let } x=n^{\prime} \text { in } t^{\prime}
\end{aligned}
$$

(by induction hypothesis)

Conclusion We have proved computational completeness of the focused logic: for any $\Gamma, \Delta \vdash t: A$, there exists some $\Gamma ; \Delta \vdash_{\text {inv }}$ $t^{\prime}: A$ (such that $\Gamma ; \Delta \vdash \mathrm{NF}_{\beta}(t) \rightsquigarrow t^{\prime}: A$ ) with $t={ }_{\beta \eta} t^{\prime}$.

\section{B.2 Saturated focusing}

To prove the main theorems on saturating focused logic, we need to describe how to convert a simply-typed lambda-term (or at least a lambda-term for the focused logic, that is in $\beta$-short weak- $\eta$ long normal-form) into a valid saturated proof derivation. This can be done either as a small-step rewrite process, or as a big-step transformation. The small-step rewrite would be very similar to the preemptive rewriting relation of Chaudhuri et al. (2008); we will here explore the alternative of a big-step transformation by defining, in Figure 11, a type-preserving translation judgments of the form $\Gamma ; \Delta \vdash_{\operatorname{sinv}} t \rightsquigarrow t^{\prime}: A$, which turns a focused term $t$ into a valid saturating focused term $t^{\prime}$.

$$
\begin{array}{ll}
\Gamma::=\operatorname{varmap}\left(N_{\mathrm{at}}\right) & \text { negative or atomic context } \\
\Delta::=\operatorname{varmap}(A) & \text { general context } \\
\frac{\Gamma ; \Delta \vdash_{\operatorname{sinv}} t \rightsquigarrow t^{\prime}: A}{\Gamma ; \Delta \vdash_{\operatorname{sinv}}(t, u) \rightsquigarrow\left(t^{\prime}, u^{\prime}\right): A * B} & \Gamma ; \Delta \vdash_{\operatorname{sinv}} u \rightsquigarrow u^{\prime}: B \\
\frac{\Gamma+\text { SINV-PAIR }}{\Gamma ;}
\end{array}
$$

REW-SINV-SUM

$$
\frac{\Gamma ; \Delta, x: A \vdash_{\text {sinv }} t \rightsquigarrow t^{\prime}: C \quad \Gamma ; \Delta, x: B \vdash_{\operatorname{sinv}} u \rightsquigarrow u^{\prime}: C}{\Gamma ; \Delta, x: A+B \vdash_{\operatorname{sinv}} \delta(x, x . t, x . u) \rightsquigarrow \delta\left(x, x . t^{\prime}, x . u^{\prime}\right): C}
$$

REW-SINV-ARR

$\frac{\Gamma ; \Delta, x: A \vdash_{\operatorname{sinv}} t \rightsquigarrow t^{\prime}: B}{\Gamma ; \Delta \vdash_{\operatorname{sinv}} \lambda x . t \rightsquigarrow \lambda x . t^{\prime}: A \rightarrow B}$

$$
\begin{aligned}
& \text { REW-SAT-INTRO } \\
& \frac{\Gamma \vdash t \rightsquigarrow t^{\prime} \Uparrow P}{\Gamma ; \emptyset \vdash_{\text {sat }} t \rightsquigarrow t^{\prime}: P}
\end{aligned}
$$$$
\text { REW-SINV-END }
$$$$
\frac{\Gamma ; \Gamma^{\prime} \vdash_{\mathrm{foc}} t \rightsquigarrow t^{\prime}: P_{\mathrm{at}}}{\Gamma ; \Gamma^{\prime} \vdash_{\text {sinv }} t \rightsquigarrow t^{\prime}: P_{\mathrm{at}}}
$$

REW-SAT

$$
\Gamma^{\prime} \neq \emptyset \quad(\bar{n}, \bar{P}) \subseteq\left\{(n, P) \mid\left(\Gamma, \Gamma^{\prime} \vdash n \Downarrow P\right)\right\}
$$$$
\forall n \in \bar{n}, n \in t \quad \Gamma, \Gamma^{\prime} ; \bar{x}: \bar{P} \vdash_{\text {sinv }} t[\bar{x} / \bar{n}] \rightsquigarrow t^{\prime}: Q_{\text {at }}
$$

$$
\Gamma ; \Gamma^{\prime} \vdash_{\text {sat }} t \rightsquigarrow \text { let } \bar{x}=\bar{n} \text { in } t^{\prime}: Q_{\text {at }}
$$

$$
\begin{aligned}
& \text { REW-SINTRO-SUM REW-SINTRO-END } \\
& \Gamma \vdash t \rightsquigarrow t^{\prime} \Uparrow A_{i} \\
& \overline{\Gamma \vdash \sigma_{i} t \rightsquigarrow \sigma_{i} t^{\prime} \Uparrow A_{1}+A_{2}} \quad \overline{\Gamma \vdash t \rightsquigarrow t^{\prime} \Uparrow N_{\text {at }}}
\end{aligned}
$$

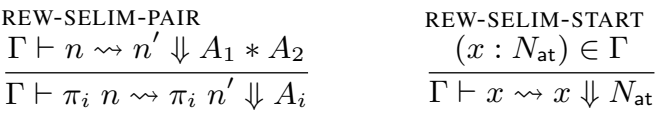

$$
\begin{aligned}
& \text { REW-SELIM-ARR } \\
& \frac{\Gamma \vdash n \rightsquigarrow n^{\prime} \Downarrow A \rightarrow B \quad \Gamma \vdash u \rightsquigarrow u^{\prime} \Uparrow A}{\Gamma \vdash n u \rightsquigarrow n^{\prime} u^{\prime} \Downarrow B} \\
& (\text { let } x=n \text { in } t)[y / n] \stackrel{\text { def }}{=} t[y / x][y / n]
\end{aligned}
$$

Figure 11. Saturation translation 
Backward search for saturated proofs corresponds to enumerating the canonical inhabitants of a given type. Our translation can be seen as a restriction of this proof search process, searching inside the $\beta \eta$-equivalence class of $t$. Because saturating proof terms are canonical (to be shown), the restricted search is deterministic modulo invertible commuting conversions.

Compared to the focusing translation of Figure 10, this rewriting is simpler as it starts from an already-focused proof whose overall structure is not affected. The only real change is moving from the left-focusing rule REW-FOC-ELIM to the saturating rule REW-SAT. Instead of allowing to cut on any neutral subterm, we enforce a maximal cut on exactly all the neutrals of $t$ that can be typed in the current environment. Because we know that "old" neutrals (those that would not satisfy the $n$ uses $\Gamma^{\prime}$ condition) have already been cut and replaced with free variables earlier in the translation, this is fact respects the using condition - except for the very first application of the rule if the initial judgment is not in the empty context, which cuts on all "old" neutrals as well.

Compared to the focusing translation, the termination of this translation is proved by structural induction - thanks to the focused structure of the input. There is but one subtlety, in the REW-SAT rule, the recursive call is on $t[\bar{x} / \bar{n}]$ which is not a subterm of the input $t$; but this rule can only happen once in a row on $t$ or its substitution: either it introduces some positives, and there must be invertible rules that recur on strict subterms (of $t[\bar{x} / \bar{n}]$, which are smallers than the strict subterms of $t$ ), or no neutrals were bound and the next invertible rules can only be REW-SAT-INTRO or REW-SAT-ATOM.

Lemma 3 (Translation soundness). If $\Gamma ; \Delta \vdash_{\text {inv }} t: A$ and $\Gamma ; \Delta \vdash_{\sin v} t \rightsquigarrow t^{\prime}:$ A then $t={ }_{\beta \eta} t^{\prime}$.

Proof. By immediate induction.

Lemma 4 (Translation validity). Suppose that $\Gamma ; \Delta \vdash_{\text {inv }} t: A$ holds in the focused logic, and that $t$ has no "old" neutral: for no $n \in t$ do we have $\Gamma \vdash n \Downarrow P$. Then, $\Gamma ; \Delta \vdash_{\text {sinv }} t \rightsquigarrow t^{\prime}:$ A implies that $\Gamma ; \Delta \vdash_{\operatorname{sinv}} t^{\prime}: A$ in the saturated focusing logic.

Proof. The restriction on "old" neutrals is necessary because the REW-SAT rule would not know what to do on such old neutrals it assumes that they were all substituted away for fresh variable in previous inference steps.

With this additional invariant the proof goes by immediate induction. In the REW-SAT rule, this invariant tells us that the bindings satisfy the freshness condition of the SAT rule of saturated logic, and because we select all such fresh bindings we preserve the property that the extended context $\Gamma, \Gamma^{\prime}$ has no old neutrals either.

Lemma 5 (Translation totality and determinism). If $\Gamma ; \Delta \vdash_{\text {inv }} t$ : $A$ there exists $a$ unique $t^{\prime}$ such that $\Gamma ; \Delta \vdash_{\text {sinv }} t \rightsquigarrow t^{\prime}: A$.

Proof. By immediate induction.

Note that the indeterminacy of invertible step ordering is still present in saturating focused logic: a non-focused term $t$ may have several saturated translations that only equal upto commuting conversions $\left(=_{\mathrm{icc}}\right)$. However, there is no more variability than in the focused proof of the non-saturating focused logic; because we translate from those, we can respect the ordering choices that are made, and the translation is thus fully deterministic.

Theorem (2: Computational completeness of saturating focused logic). If we have $\emptyset ; \Delta \vdash_{\text {inv }} t: A$ in the non-saturating focused logic, then for some $u={ }_{\beta \eta} t$ we have $\emptyset ; \Delta \vdash_{\operatorname{sinv}} u: A$ in the saturating focused logic.
Proof. This is an immediate corollary of the previous results. By totality of the translation (Lemma 5) we have a $u$ such that $\emptyset ; \Delta \vdash_{\text {sinv }}$ $t \rightsquigarrow u: A$. By validity (Lemma 4) we have that $\emptyset ; \Delta \vdash_{\text {sinv }} u: A$ in the saturating focused calculus - the condition that there be no old neutrals is trivially true for the empty context $\emptyset$. Finally, by soundness (Lemma 3) we have that $\operatorname{let} \exp (t)={ }_{\beta \eta} l \operatorname{let} \exp (u)$.

Lemma 6 (Determinacy of saturated translation). For any $u_{1}, u_{2}$, if we have $\Gamma ; \Delta \vdash_{\text {inv }} t \rightsquigarrow u_{1}: A$ and $\Gamma ; \Delta \vdash_{\text {inv }} t \rightsquigarrow u_{2}:$ A then we have $\Gamma ; \Delta \vdash_{\text {sinv }} u_{1} \rightsquigarrow r_{1}: A$ and $\Gamma ; \Delta \vdash_{\operatorname{sinv}} u_{2} \rightsquigarrow r_{2}: A$ with $r_{1}={ }_{\text {icc }} r_{2}$.

Proof. There are only two sources of non-determinism in the focused translation:

- an arbitrary choice of the order in which to apply the invertible rules

- a neutral let-extrusion may happen at any point between the first scope where it is well-defined to the lowest common ancestors of all uses of the neutral in the term.

The first source of non-determinism gives $(=$ icc $)$-equivalent derivations. The second disappears when doing the saturating translation, which enforces a unique placement of let-extrusions at the first scope where the strictly positive neutrals are well-defined.

As a result, two focused translations of the same term may differ in both aspect, but their saturated translations differ at most by $\left(={ }_{\text {icc }}\right)$.

Definition 1 (Normalization by saturation). For a well-typed (nonfocused) $\lambda$-term $\Gamma, \Delta \vdash t: A$, we write $\mathrm{NF}_{\text {sat }}(t)$ for any saturated term $t^{\prime \prime}$ such that

$$
\Gamma ; \Delta \vdash_{\text {inv }} \mathrm{NF}_{\beta}(t) \rightsquigarrow t^{\prime}: A \quad \Gamma ; \Delta \vdash_{\text {sinv }} t^{\prime} \rightsquigarrow t^{\prime \prime}: A
$$

Note that all possible $t^{\prime \prime}$ are equal modulo $\left(={ }_{\mathrm{icc}}\right)$, by the Determinacy Lemma 6.

Lemma 7 (Saturation congruence). For any context $C[\square]$ and term $t$ we have

$$
\mathrm{NF}_{\text {sat }}(C[t])={ }_{\mathrm{icc}} \mathrm{NF}_{\text {sat }}\left(C\left[\mathrm{NF}_{\text {sat }}(t)\right]\right)
$$

Proof. We reason by induction on $C[\square]$. Without loss of generality we will assume $C[\square]$ atomic. It is either a redex-forming context

$$
\square u \quad \pi_{k} \square \quad \delta\left(\square, x . u_{1}, x . u_{2}\right)
$$

or a non-redex forming context

$$
\begin{array}{cr}
u \square & \sigma_{i} \square \\
(u, \square) & (\square, u) \\
\delta\left(u, x . \square, x . u_{2}\right) & \delta\left(u, x . u_{1}, x . \square\right)
\end{array}
$$

If it is a non-context-forming redex, then we have $\mathrm{NF}_{\beta}(C[t])=$ $C\left[\mathrm{NF}_{\beta}(t)\right]$. The focused and saturated translations then work over $\left.C\left[\mathrm{NF}_{\beta}(t)\right]\right]$ just as they work with $\mathrm{NF}_{\beta}(t)$, possibly adding bindings before $C[\square]$ instead of directly on the (translations of) $\mathrm{NF}_{\beta}(t)$. The results are in the $\left(=_{\mathrm{icc}}\right)$ relation.

The interesting case is when $C[\square]$ is a redex-forming context: a reduction may overlap the frontier between $C[\square]$ and the plugged term. In that case, we will reason on the saturated normal form $\mathrm{NF}_{\text {sat }}(t)$. Thanks to the strongly restricted structure of focused and saturated normal form, we have precise control over the possible reductions. 
Application case $C[\square] \stackrel{\text { def }}{=} \square u$. We prove that there exist $t^{\prime}$ such that $\Gamma ; \Delta \vdash_{\text {inv }} t \rightsquigarrow t^{\prime}: A \rightarrow B$, and a $r$ such that both $\Gamma ; \Delta \vdash_{\text {inv }} t u \rightsquigarrow r: B$ and $\Gamma ; \Delta \vdash_{\text {inv }} t^{\prime} u \rightsquigarrow r: B$ hold. This implies the desired result - after translation of $r$ into a saturated term. The proof proceeds by induction on the derivation $\Gamma ; \Delta \vdash_{\text {inv }} t u \rightsquigarrow r: B$ (we know that all possible such translations have finite derivations).

To make the proof easier to follow, we introduce the notation $\mathrm{NF}_{\text {foc }}(\Gamma ; \Delta \vdash t)$ to denote a focused translation $t^{\prime}$ of $\mathrm{NF}_{\beta}(t)$ (that is, $\Gamma ; \Delta \vdash_{\text {inv }} t \rightsquigarrow t^{\prime}: A$, where $A$ is uniquely defined by $\left.\Gamma ; \Delta \vdash_{\text {inv }} t^{\prime}: A\right)$ ). This notation should be used with care because it is not well-determined: there are many such possible translations. Statements using the notation should be interpreted existentially: $P\left(\mathrm{NF}_{\mathrm{foc}}(\Gamma ; \Delta \vdash t)\right)$ means that there exists a translation $t^{\prime}$ of $t$ such that $P\left(t^{\prime}\right)$ holds. The current goal (whose statement took the full previous paragraph) can be rephrased as follows:

$$
\mathrm{NF}_{\text {foc }}(\Gamma ; \Delta \vdash t u)=\mathrm{NF}_{\text {foc }}\left(\Gamma ; \Delta \vdash \mathrm{NF}_{\text {foc }}(\Gamma ; \Delta \vdash t) u\right)
$$

We will simply write $\mathrm{NF}_{\text {foc }}(t)$ when the typing environment of the translation is clear from the context.

If $\Delta$ contains a strictly positive type, it is of the form $\left(\Delta^{\prime}, x\right.$ : $C_{1}+C_{2}$ ) and we can get by induction hypothesis that

$$
\mathrm{NF}_{\text {foc }}\left(\Gamma ; \Delta^{\prime}, x: C_{i} \vdash t u\right)=\mathrm{NF}_{\text {foc }}\left(\Gamma ; \Delta^{\prime}, x: C_{i} \vdash \mathrm{NF}_{\text {foc }}(t) u\right)
$$

for $i$ in $\{1,2\}$, from which we can conclude with

$$
\begin{aligned}
& \mathrm{NF}_{\mathrm{foc}}\left(\Gamma ; \Delta^{\prime}, x: C_{1}+C_{2} \vdash t u\right) \\
= & \delta\left(x, x \cdot \mathrm{NF}_{\mathrm{foc}}\left(\Gamma ; \Delta^{\prime}, x: C_{1} \vdash t u\right), x \ldots C_{2} \ldots\right) \\
= & \delta\left(x, x \cdot \mathrm{NF}_{\mathrm{foc}}\left(\Gamma ; \Delta^{\prime}, x: C_{1} \vdash \mathrm{NF}_{\mathrm{foc}}(t) u\right), x \ldots C_{2} \ldots\right) \\
= & \mathrm{NF}_{\mathrm{foc}}\left(\Gamma ; \Delta^{\prime}, x: C_{1}+C_{2} \vdash \mathrm{NF}_{\mathrm{foc}}(t) u\right)
\end{aligned}
$$

Otherwise $\Delta$ is a negative or atomic context.

Any focused translation of $t$ at type $A \rightarrow B$ is thus necessarily of the form $\lambda x . \mathrm{NF}_{\text {foc }}(t x)$. In particular, any $\mathrm{NF}_{\text {foc }}\left(\mathrm{NF}_{\text {foc }}(t) u\right)$, that is, any $\mathrm{NF}_{\text {foc }}\left(\left(\lambda x \cdot \mathrm{NF}_{\text {foc }}(t x)\right) u\right)$, is equal by stability of the translation to $\beta$-reduction to a term of the form $\mathrm{NF}_{\text {foc }}\left(\mathrm{NF}_{\mathrm{foc}}(t x)[u / x]\right)$. On the other hand, $\mathrm{NF}_{\text {foc }}(t u)$ can be of several different forms.

Note that $t u$ is translated at the same type as $t x$. In particular, if this is a negative type, they both begin with a suitable $\eta$-expansion (of a product or function type); in the product case for example, we have $\mathrm{NF}_{\text {foc }}(t u)=\left(\mathrm{NF}_{\text {foc }}\left(\pi_{1}\left(\begin{array}{ll}t & u\end{array}\right)\right), \mathrm{NF}_{\text {foc }}\left(\pi_{2}\left(\begin{array}{ll}t & u\end{array}\right)\right)\right)$, and similarly $\mathrm{NF}_{\text {foc }}(t x)=\left(\mathrm{NF}_{\mathrm{foc}}\left(\pi_{1}(t x)\right), \mathrm{NF}_{\mathrm{foc}}\left(\pi_{2}(t x)\right)\right)$ : we can then conclude by induction hypothesis on those smaller pairs of terms $\pi_{i}(t u)$ and $\pi_{i}(t x)$ for $i$ in $\{1,2\}$. We can thus assume that $t u$ is of positive or atomic type, and will reason by case analysis on the $\beta$-normal form of $t$.

If $\mathrm{NF}_{\beta}(t)$ is of the form $\lambda x . t^{\prime}$ for some $t^{\prime}$, then $\mathrm{NF}_{\text {foc }}(t u)$ is equal to $\mathrm{NF}_{\text {foc }}\left(\left(\lambda x \cdot t^{\prime}\right) u\right)$, that is, $\mathrm{NF}_{\text {foc }}\left(t^{\prime}[u / x]\right)$. Finally, we have $\mathrm{NF}_{\text {foc }}(t x)=\mathrm{NF}_{\text {foc }}\left(\left(\lambda x \cdot t^{\prime}\right) x\right)=\mathrm{NF}_{\text {foc }}\left(t^{\prime}\right)$, which allows to conclude from our assertion that $\mathrm{NF}_{\text {foc }}\left(\mathrm{NF}_{\text {foc }}(t) u\right)$ is equal to $\mathrm{NF}_{\text {foc }}\left(\mathrm{NF}_{\text {foc }}(t x)[u / x]\right)$.

If $\mathrm{NF}_{\beta}(t)$ contains a strictly positive neutral subterm $n: P$ (this is in particular always the case when it is of the form $\delta\left(t^{\prime}, \ldots\right)$ ), we can let-extrude it to get

$$
\begin{gathered}
\mathrm{NF}_{\text {foc }}\left(\Gamma ; \Gamma^{\prime} \vdash t\right) \\
=\text { let } x=\mathrm{NF}_{\text {foc }}(n) \text { in } \operatorname{NF}_{\text {foc }}\left(\Gamma, \Gamma^{\prime} ; x: P \vdash t[x / n]\right)
\end{gathered}
$$

But then $n: P$ is also a strictly positive neutral subterm of let $x=\mathrm{NF}_{\text {foc }}(n)$ in $\mathrm{NF}_{\text {foc }}(t[x / n])$, so we have

$$
\begin{aligned}
& \mathrm{NF}_{\mathrm{foc}}\left(\mathrm{NF}_{\mathrm{foc}}(t) u\right) \\
= & \mathrm{NF}_{\mathrm{foc}}\left(\text { let } x=\mathrm{NF}_{\mathrm{foc}}(n) \text { in } \mathrm{NF}_{\mathrm{foc}}(t[x / n])\right) \\
= & \text { let } x=\mathrm{NF}_{\mathrm{foc}}(n) \text { in }_{\mathrm{NF}} \text { foc }(t[x / n])[x / n] \\
= & \text { let } x=\mathrm{NF}_{\mathrm{foc}}(n) \text { in } \mathrm{NF}_{\mathrm{foc}}(t[x / n]) \\
= & \mathrm{NF}_{\mathrm{foc}}(t u)
\end{aligned}
$$

Finally, if $\mathrm{NF}_{\beta}(t)$ contains no strictly positive neutral subterm, the rule REW-UP-ARROW applies: $\mathrm{NF}_{\mathrm{foc}}(t u)$ is of the form $n \mathrm{NF}_{\text {foc }}(u)$, where $n \stackrel{\text { def }}{=} \mathrm{NF}_{\text {foc }}(t)$. In this case we also have $\mathrm{NF}_{\text {foc }}(t x)=n x$, and thus

$$
\begin{array}{ll}
= & \mathrm{NF}_{\mathrm{foc}}\left(\mathrm{NF}_{\mathrm{foc}}(t) x\right) \\
= & \mathrm{NF}_{\mathrm{foc}}\left(\mathrm{NF}_{\mathrm{foc}}(t x) x u[\right. \\
= & \mathrm{NF}_{\mathrm{foc}}(n u) \\
& \mathrm{NF}_{\mathrm{foc}}(t u)
\end{array}
$$

Projection case $C[\square] \stackrel{\text { def }}{=} \pi_{i} \square$ The case $C[\square] \stackrel{\text { def }}{=} \pi_{i} t$ is proved in the same way as the application case: after some sum eliminations, the translation of $t$ is an $\eta$-expansion of the product, which is related to the translations $\mathrm{NF}_{\text {foc }}\left(\pi_{i} t\right)$, which either reduce the product or build a neutral term $\pi_{i} n$ after introducing some let-bindings.

Sum elimination case $C[\square] \stackrel{\text { def }}{=} \delta\left(\square, x . u_{1}, x . u_{2}\right) \quad$ Reusing the notations of the application case, show that

$$
\mathrm{NF}_{\text {foc }}\left(\delta\left(t, x . u_{1}, x . u_{2}\right)\right)=\mathrm{NF}_{\text {foc }}\left(\delta\left(\mathrm{NF}_{\text {foc }}(t), x . u_{1}, x . u_{2}\right)\right)
$$

In the case of the function application or pair projection, the congruence proof uses the fact that the translation of $t$ (of function or product type) necessarily starts with a $\lambda$-abstraction or pair construction - in fact, we follow the incremental construction of the first invertible phase, in particular we start by eliminating sums from the context.

In the case of the sum elimination, we must follow the translation into focused form further: we know the first invertible phase of $\mathrm{NF}_{\mathrm{foc}}(t)$ may only have sum-eliminations (pair or function introductions would be ill-typed as $t$ has a sum type $A+B$ ).

As in the application case, we can then extrude neutrals from $t$, and the extrusion can be mirrored in both $\mathrm{NF}_{\mathrm{foc}}(\delta(t, \ldots))$ and $\mathrm{NF}_{\text {foc }}\left(\delta\left(\mathrm{NF}_{\text {foc }}(t), \ldots\right)\right)$. Finally, we reason by case analysis on $\mathrm{NF}_{\beta}(t)$.

If $\mathrm{NF}_{\beta}(t)$ is of the form $\sigma_{i} t^{\prime}$, then we have

$$
\begin{aligned}
& \mathrm{NF}_{\text {foc }}\left(\delta\left(\mathrm{NF}_{\text {foc }}(t), x . u_{1}, x . u_{2}\right)\right) \\
= & \mathrm{NF}_{\text {foc }}\left(\delta\left(\sigma_{i} \mathrm{NF}_{\text {foc }}\left(t^{\prime}\right), x \cdot u_{1}, x . u_{2}\right)\right) \\
= & \mathrm{NF}_{\text {foc }}\left(u_{i}\left[\mathrm{NF}_{\text {foc }}\left(t^{\prime}\right) / x\right]\right)
\end{aligned}
$$

and

$$
\begin{aligned}
& \mathrm{NF}_{\text {foc }}\left(\delta\left(t, x . u_{1}, x . u_{2}\right)\right) \\
= & \mathrm{NF}_{\text {foc }}\left(\delta\left(\mathrm{NF}_{\beta}(t), x . u_{1}, x . u_{2}\right)\right) \\
= & \mathrm{NF}_{\text {foc }}\left(\delta\left(\sigma_{i} t^{\prime}, x . u_{1}, x . u_{2}\right)\right) \\
= & \mathrm{NF}_{\text {foc }}\left(u_{i}\left[t^{\prime} / x\right]\right)
\end{aligned}
$$

What is left to prove is that $\mathrm{NF}_{\text {foc }}\left(u_{i}\left[\mathrm{NF}_{\text {foc }}\left(t^{\prime}\right) / x\right]\right)=\mathrm{NF}_{\text {foc }}\left(u_{i}\left[t^{\prime} / x\right]\right)$ but that is equivalent (by stability of the focusing translation by $\beta$ reduction) to $\mathrm{NF}_{\text {foc }}\left(\left(\lambda x . u_{i}\right) \mathrm{NF}_{\text {foc }}\left(t^{\prime}\right)\right)=\mathrm{NF}_{\text {foc }}\left(\left(\lambda x . u_{i}\right) t^{\prime}\right)$, which is exactly the application case proved previously.

This is in fact the only possible case: when all strictly positive neutrals have been extruded, then $\mathrm{NF}_{\beta}(t)$ is necessarily an injection $\sigma_{i} t^{\prime}$ (already handled) or a variable $x$ (this corresponds to the case where $t$ itself reduces to a strictly positive neutral), but this variable would be in the context and of strictly positive type, so this case is already handled as well.

Theorem (1: Canonicity of saturating focused logic). If we have $\Gamma ; \Delta \vdash_{\operatorname{sinv}} t: A$ and $\Gamma ; \Delta \vdash_{\operatorname{sinv}} u: A$ in saturating focused logic with $t \neq_{\text {icc }} u$, then $t \neq_{\beta \eta} u$.

Proof. By contrapositive: if $t={ }_{\beta \eta} u$ (that is, if $\operatorname{let} \exp (t)={ }_{\beta \eta}$ let $\exp (u)$ ) then $t={ }_{\text {icc }} u$.

The difficulty to prove this statement is that $\beta \eta$-equivalence does not preserve the structure of saturated proofs: an equivalence proof may go through intermediate steps that are neither saturated nor focused or in $\beta$-normal form. 
We will thus go through an intermediate relation, which we will write $\left(\sim_{\text {sat }}\right)$, defined as follows on arbitrary well-typed lambdaterms:

$$
\begin{aligned}
& \emptyset ; \Delta \vdash_{\text {inv }} t: A \quad \emptyset ; \Delta \vdash_{\text {inv }} u: A \\
& \emptyset ; \Delta \vdash_{\text {inv }} \mathrm{NF}_{\beta}(t) \rightsquigarrow t^{\prime}: A \quad \emptyset ; \Delta \vdash_{\text {inv }} \mathrm{NF}_{\beta}(u) \rightsquigarrow u^{\prime}: A \\
& \emptyset ; \Delta \vdash_{\operatorname{sinv}} t^{\prime} \rightsquigarrow t^{\prime \prime}: A \quad \emptyset ; \Delta \vdash_{\operatorname{sinv}} u^{\prime} \rightsquigarrow u^{\prime \prime}: A \\
& t^{\prime \prime}={ }_{\text {icc }} u^{\prime \prime} \\
& \Delta \vdash t \sim_{\text {sat }} u: A
\end{aligned}
$$

It follows from the previous results that if $t \sim_{\text {sat }} u$, then $t={ }_{\beta \eta} u$. We will now prove the converse inclusion: if $t={ }_{\beta \eta} u$ (and they have the same type), then $t \sim_{\text {sat }} u$ holds. In the particular case of terms that happen to be (let-expansions of) valid saturated focused derivations, this will tell us in particular that $t={ }_{\text {icc }} u$ holds - the desired result.

The computational content of this canonicity proof is an equivalence algorithm: $\left(\sim_{\text {sat }}\right)$ is a decidable way to check for $\beta \eta$ equality, by normalizing terms to their saturated (or maximally multi-focused) structure.

$\beta$-reductions It is immediate that $\left(=_{\beta}\right)$ is included in $\left(\sim_{\text {sat }}\right)$. Indeed, if $t=\beta u$ then $\mathrm{NF}_{\beta}(t)=\mathrm{NF}_{\beta}(u)$ and $t \sim_{\text {sat }} u$ is trivially satisfied.

Negative $\eta$-expansions We can prove that if $t={ }_{\eta} u$ through one of the equations

$$
(t: A \rightarrow B)={ }_{\eta} \lambda x . t x \quad(t: A * B)={ }_{\eta}\left(\pi_{1} t, \pi_{2} t\right)
$$

then both $t$ and $u$ are rewritten in the same focused proof $r$. We have both $\emptyset ; \Delta \vdash_{\text {inv }} t \rightsquigarrow r: A$ and $\emptyset ; \Delta \vdash_{\text {inv }} u \rightsquigarrow r: A$, and thus $t \sim_{\text {sat }} u$. Indeed we have:

$$
\begin{gathered}
\frac{\emptyset ; \Delta, x: A \vdash_{\mathrm{inv}} \mathrm{NF}_{\beta}(t x) \rightsquigarrow r^{\prime}: B}{\emptyset ; \Delta \vdash_{\mathrm{inv}} t \rightsquigarrow \lambda x . r^{\prime}: A \rightarrow B} \\
\frac{\mathrm{NF}_{\beta}((\lambda x . t x) x)=\mathrm{NF}_{\beta}(t x)}{\emptyset ; \Delta: A \vdash_{\mathrm{inv}} \mathrm{NF}_{\beta}((\lambda x . t x) x) \rightsquigarrow r^{\prime}: B} \\
\emptyset ; \Delta \vdash_{\mathrm{inv}} \lambda x . t x \rightsquigarrow \lambda x . r^{\prime}: A \rightarrow B
\end{gathered}
$$

and

$$
\begin{gathered}
\frac{\forall i \in\{1,2\}, \quad \emptyset ; \Delta \vdash_{\mathrm{inv}} \mathrm{NF}_{\beta}\left(\pi_{i} t\right) \rightsquigarrow r_{i}^{\prime}: A_{i}}{\emptyset ; \Delta \vdash_{\mathrm{inv}} t \rightsquigarrow\left(r_{1}^{\prime}, r_{2}^{\prime}\right):\left(A_{1}, A_{2}\right)} \\
\frac{\pi_{i}\left(\pi_{1} t_{1}, \pi_{2} t_{2}\right)=t_{i}}{\emptyset i \in\{1,2\}, \quad \emptyset ; \Delta \vdash_{\mathrm{inv}} \mathrm{NF}_{\beta}\left(\pi_{i}\left(\pi_{1} t_{1}, \pi_{2} t_{2}\right)\right) \rightsquigarrow r_{i}^{\prime}: A_{i}} \\
\emptyset ; \Delta \vdash_{\mathrm{inv}}\left(\pi_{1} t_{1}, \pi_{2} t_{2}\right) \rightsquigarrow\left(r_{1}^{\prime}, r_{2}^{\prime}\right):\left(A_{1}, A_{2}\right)
\end{gathered}
$$

Positive $\eta$-expansion The interesting case is the positive $\eta$ expansion

$$
\forall C[\square], \quad C[t: A+B]={ }_{\eta} \delta\left(t, x . C\left[\sigma_{1} x\right], x . C\left[\sigma_{2} x\right]\right)
$$

We do a case analysis on the (weak head) $\beta$-normal form of $t$. If it is an injection of the form $\sigma_{i} t^{\prime}$, then the equation becomes true by a simple $\beta$-reduction:

$$
\delta\left(\sigma_{i} t^{\prime}, x . C\left[\sigma_{1} x\right], x . C\left[\sigma_{2} x\right]\right) \rightsquigarrow \beta C\left[\sigma_{i} t^{\prime}\right]
$$

Otherwise the $\beta$-normal form of $t$ is a positive term that does not start with an injection. In particular, $\mathrm{NF}_{\beta}(t)$ is not reduced when reducing the whole term $C[t]$ (only possibly duplicated): for some multi-hole context $C^{\prime}$ we have $\mathrm{NF}_{\beta}(C[t])=C^{\prime}\left[\mathrm{NF}_{\beta}(t)\right]$ and

$$
\begin{aligned}
& \mathrm{NF}_{\beta}\left(\delta\left(t, x . C\left[\sigma_{1} x\right], x . C\left[\sigma_{2} x\right]\right)\right) \\
= & \delta\left(\mathrm{NF}_{\beta}(t), x \cdot C^{\prime}\left[\sigma_{1} x\right], x \cdot C^{\prime}\left[\sigma_{2} x\right]\right)
\end{aligned}
$$

Without loss of generality, we can assume that $\mathrm{NF}_{\beta}(t)$ is a neutral term. Indeed, if it is not, it starts with a (possibly empty) series of non-invertible elimination forms, applied to a sum-elimination construction - which is itself either a neutral or of this form. It eventually contains a neutral strict subterm of strictly positive type valid in the current scope. The focused translation can then cut on this strictly positive neutral, split on the sum type, and replace the neutral with either $\sigma_{1} z$ or $\sigma_{2} z$ for some fresh $z$. This can be done on both terms equated by the $\eta$-equivalence for sums, and returns (two pairs of) $\eta$-equivalent terms with one less strictly possible neutral strict subterm.

Let $n \stackrel{\text { def }}{=} \mathrm{NF}_{\beta}(t)$. It remains to show that the translations of $C^{\prime}[n]$ and $\delta\left(n, x . C^{\prime}\left[\sigma_{1} x\right], x . C^{\prime}\left[\sigma_{2} x\right]\right)$ are equal modulo invertible commuting conversions. In fact, we show that they translate to the same focused proof:

$$
\begin{gathered}
\Gamma \vdash n: A+B \quad \Gamma \vdash_{\text {ne }} n \rightsquigarrow n^{\prime} \Downarrow A+B \\
\Gamma ; x: A \vdash_{\text {inv }} C^{\prime}\left[\sigma_{1} x\right] \rightsquigarrow r_{1}: D \\
\Gamma ; x: B \vdash_{\text {inv }} C^{\prime}\left[\sigma_{2} x\right] \rightsquigarrow r_{2}: D \\
\frac{\Gamma ; x: A+B \vdash_{\text {inv }} C^{\prime}[x] \rightsquigarrow \delta\left(x, x \cdot r_{1}, x \cdot r_{2}\right): D}{\Gamma \vdash_{\text {foc }} C^{\prime}[n] \rightsquigarrow \operatorname{let} x=n \text { in } \delta\left(x, x \cdot r_{1}, x \cdot r_{2}\right): D} \\
\Gamma \vdash n: A+B \quad \Gamma \vdash_{\text {ne }} n \rightsquigarrow n^{\prime} \Downarrow A+B \\
\operatorname{NF}_{\beta}\left(\delta\left(\sigma_{i} x x \cdot C^{\prime}\left[\sigma_{1} x\right] x \cdot C^{\prime}\left[\sigma_{2} x\right],\right),=\right) C^{\prime}\left[\sigma_{i} x\right] \\
\Gamma ; x: A \vdash_{\text {inv }} C^{\prime}\left[\sigma_{1} x\right] \rightsquigarrow r_{1}: D \\
\Gamma ; x: B \vdash_{\text {inv }} C^{\prime}\left[\sigma_{2} x\right] \rightsquigarrow r_{2}: D \\
\frac{\Gamma ; x: A+B \vdash_{\text {inv }} \delta\left(x, x \cdot C^{\prime}\left[\sigma_{1} x\right], x \cdot C^{\prime}\left[\sigma_{2} x\right]\right) \rightsquigarrow \delta\left(x, x \cdot r_{1}, x . r_{2}\right): D}{\Gamma \vdash_{\text {foc }} \delta\left(n, x \cdot C^{\prime}\left[\sigma_{1} x\right], x \cdot C^{\prime}\left[\sigma_{2} x\right]\right) \rightsquigarrow \operatorname{let} x=n \text { in } \delta\left(x, x . r_{1}, x \cdot r_{2}\right): D}
\end{gathered}
$$

Transitivity Given $t \sim_{\text {sat }} u$ and $u \sim_{\text {sat }} r$, do we have $t \sim_{\text {sat }} r$ ? In the general case we have

$$
\begin{aligned}
& \emptyset ; \Delta \vdash_{\text {inv }} t: A \quad \emptyset ; \Delta \vdash_{\text {inv }} u: A \\
& \emptyset ; \Delta \vdash_{\text {inv }} \mathrm{NF}_{\beta}(t) \rightsquigarrow t^{\prime}: A \quad \emptyset ; \Delta \vdash_{\text {inv }} \mathrm{NF}_{\beta}(u) \rightsquigarrow u_{1}^{\prime}: A \\
& \emptyset ; \Delta \vdash_{\text {sinv }} t^{\prime} \rightsquigarrow t^{\prime \prime}: A \quad \emptyset ; \Delta \vdash_{\text {sinv }} u_{1}^{\prime} \rightsquigarrow u_{1}^{\prime \prime}: A \\
& t^{\prime \prime}={ }_{\text {icc }} u_{1}^{\prime \prime} \\
& \Delta \vdash t \sim_{\text {sat }} u: A \\
& \emptyset ; \Delta \vdash_{\text {inv }} u: A \quad \emptyset ; \Delta \vdash_{\text {inv }} r: A \\
& \emptyset ; \Delta \vdash_{\text {inv }} \mathrm{NF}_{\beta}(u) \rightsquigarrow u_{2}^{\prime}: A \quad \emptyset ; \Delta \vdash_{\text {inv }} \mathrm{NF}_{\beta}(r) \rightsquigarrow r^{\prime}: A \\
& \emptyset ; \Delta \vdash_{\text {sinv }} u_{2}^{\prime} \rightsquigarrow u_{2}^{\prime \prime}: A \quad \emptyset ; \Delta \vdash_{\text {sinv }} r^{\prime} \rightsquigarrow r^{\prime \prime}: A \\
& u_{2}^{\prime \prime}={ }_{\text {icc }} r^{\prime \prime} \\
& \Delta \vdash u \sim_{\text {sat }} r: A
\end{aligned}
$$

By determinacy of the saturating translation (Lemma 6) we have that $u_{1}^{\prime \prime}={ }_{\text {icc }} u_{2}^{\prime \prime}$. Then, by transitivity of $(=$ icc $)$ :

$$
t^{\prime \prime}==_{\text {icc }} u_{1}^{\prime \prime}={ }_{\text {icc }} u_{2}^{\prime \prime}={ }_{\text {icc }} r^{\prime \prime}
$$

Congruence If $\Delta \vdash t_{1} \sim_{\text {sat }} t_{2}: A$, do we have that $C\left[t_{1}\right] \sim_{\text {sat }}$ $C\left[t_{2}\right]$ for any term context $C$ ?

This is an immediate application of the Saturation Congruence Lemma (7): it tells us that $\mathrm{NF}_{\text {sat }}\left(C\left[t_{1}\right]\right)=$ icc $\mathrm{NF}_{\text {sat }}\left(C\left[\mathrm{NF}_{\text {sat }}\left(t_{1}\right)\right]\right)$ and $\mathrm{NF}_{\text {sat }}\left(C\left[t_{1}\right]\right)={ }_{\text {icc }} \mathrm{NF}_{\text {sat }}\left(C\left[\mathrm{NF}_{\text {sat }}\left(t_{2}\right)\right]\right)$. So, by transitivity of $(=$ icc $)$ we only have to prove $\mathrm{NF}_{\text {sat }}\left(C\left[\mathrm{NF}_{\text {sat }}\left(t_{1}\right)\right]\right)=$ icc $\mathrm{NF}_{\text {sat }}\left(C\left[\mathrm{NF}_{\text {sat }}\left(t_{1}\right)\right]\right)$, which is a consequence of our assumption $\mathrm{NF}_{\text {sat }}\left(t_{1}\right)={ }_{\text {icc }} \mathrm{NF}_{\text {sat }}\left(t_{2}\right)$ and congruence of $\left(=_{\text {icc }}\right)$.

\section{B.3 Two-or-more counting}

The full proofs, and additional results, are available as a research report (Scherer 2014).

\section{B.4 Algorithm}

The algorithm is described as a system of inference rules in Figure 9.

Lemma 8 (Termination). The algorithmic inference system only admits finite derivations. 
Proof. We show that each inference rule is of finite degree (it has a finite number of premises), and that there exists no infinite path of inference rules - concluding with König's Lemma.

Degree finiteness The rules that could be of infinite degree are ALG-SAT (which quantifies over all positives $P$ ) and ALG-SELIM (which quantifies over arbitrarily elimination derivations). But both rules have been restricted through the subformula property to only quantify on finitely many formulas (ALG-SAT) or possible elimination schemes (ALG-SELIM).

Infinite paths lead to absurdity We first assert that any given phase (invertible, saturation, introductions/eliminations) may only be of finite length. Indeed, invertible rules have either the context or the goal decreasing structurally. Saturation rules are either ALG-SAT if $\Gamma^{\prime} \neq \emptyset$, which is immediately followed by elimination and invertible rules, or ALG-SAT-KILL or ALG-SAT-POST if $\Gamma^{\prime}=\emptyset$, in which case the derivation either terminates or continues with a noninvertible introduction or elimination. Introductions have the goal decreasing structurally, and eliminations have the goal increasing structurally, and can only form valid derivations if it remains a subformula of the context $\Gamma$.

Given that any phase is finite, any infinite path will necessarily have an infinite number of phase alternation. By looking at the graph of phase transitions (invertible goes to saturating which goes to introductions or eliminations, which go to invertible), we see that each phase will occur infinitely many times along an infinite path. In particular, an infinite path would have infinitely many invertible and saturation phases; the only transition between them is the rule ALG-SINV-END which must occur infinitely many times in the path.

Now, because the rules grow the context monotonically, an infinite path must eventually reach a maximal stable context $\Gamma$, that never grows again along the path. In particular, for infinitely many ALG-SINV-END we have $\Gamma$ maximal and thus $\Gamma^{\prime}-{ }_{2} \Gamma=\emptyset-$ if the trimming was not empty, $\Gamma^{\prime}$ would grow strictly after the next saturation phase, while we assumed it was maximal.

This means that either ALG-SAT-KILL or ALG-SAT-POST incurs infinitely many times along the infinite path. Those rules check the memory count of the current (context, goal) pair $\Gamma \vdash P$. Because of the subformula property (formulas occurring in subderivations are subformulas of the root judgment concluding the complete proof), there can be only finitely many different $\Gamma \vdash P$ pair ( $\Gamma$ is a 2 mapping which grows monotonically).

An infinite path would thus necessarily have infinitely many steps ALG-SAT-KILL or ALG-SAT-POST with the same (context, goal) pair. This is impossible, as a given pair can only go at most twice through ALG-SAT-POST, and going through ALG-SAT-KILL terminates the path. There is no infinite path.

Lemma 9 (Totality and Determinism). For any algorithmic judgment there is exactly one applicable rule.

Proof. Immediate by construction of the rules. Invertible rules $M @ \Gamma ; \Gamma^{\prime} ; \Sigma \vdash_{\text {inv }}^{\text {alg }} S: A$ are directed by the shape of the context $\Sigma$ and the goal $A$. Saturation rules $M @ \Gamma ; \Gamma^{\prime} \vdash_{\text {sat }}^{\text {alg }} S: A$ are directed by the new context $\Gamma^{\prime}$. If $\Gamma^{\prime}=\emptyset$, the memory $M(\Gamma \vdash A)$ decides whether to kill or post-saturate, in which case the shape of the goal (either strict positive or atomic) directs the post-saturation rule. Finally, non-invertible introductions $M @ \Gamma \vdash^{\text {alg }} S \Uparrow A$ are directed by the goal $A$, and there is exactly one non-invertible elimination rule.

Note that the choice we made to restrict the ordering of invertible rules is not necessary - we merely wanted to demonstrate an example of such restrictions, and reflect the OCaml implementation. We could keep the same indeterminacy as in previous sys- tems; totality would be preserved (all judgments have one applicable rule), but determinism dropped. There could be several $S$ such that $M @ \Gamma ; \Gamma^{\prime} ; \Sigma \vdash_{\text {inv }}^{\text {alg }} S: A$, which would correspond to (2set restrictions of) sets of terms equal upto invertible commuting conversion.

Lemma 10 (Strengthening of saturated derivations). If $\Gamma \oplus\left(N_{\mathrm{at}} \mapsto\right.$ $\left.\{x\}_{2}\right) ; \Gamma^{\prime} \vdash_{\text {sat }} t: P_{\text {at }}$ or $\Gamma ; \Gamma^{\prime} \oplus\left(N_{\text {at }} \mapsto\{x\}_{2}\right) \vdash_{\text {sat }} t: P_{\text {at }}$ but $\neg(t$ uses $x)$, then $\Gamma ; \Gamma^{\prime} \vdash_{\text {sat }} t: P_{\text {at }}$ already holds.

Proof. By induction, this is immediate for all rules except SAT. In this case we must note that the set of deducible positives $\Gamma, \Gamma^{\prime} \vdash$ $n \Downarrow P$ is unchanged after strengthening, as by hypothesis we know that each $n$ does not use $x$ and can thus already be defined in the strengthened context.

Lemma 11 (Soundness). For any algorithmic judgment returning a 2-set $S$, any element $t \in S$ is a valid proof term of the corresponding saturating judgment.

Proof. By induction, this is immediate for all rules except ALG-SAT. This rule is designed to fit the requirements of the saturated logic SAT rule; the one subtlety is the fact that all inhabitants $S$ are searched in the full context $\Gamma, \Gamma^{\prime} ; \emptyset ; B$ (where $B$ binds all neutrals reachable by saturation), and then each $t \in S$ of them is implicitly strengthened to be typed only in the subset of $B$ actually used in $t$. This strengthening preserves the validity of the saturated proof, by by Lemma 10 .

Definition 2 (Recurrent ancestors). Consider a complete algorithmic derivation of a judgment with empty initial memory $\emptyset$. Given any subderivation $P_{\text {above }}$, we call recurrent ancestor any other subderivation $P_{\text {below }}$ that is on the path between $P_{\text {above }}$ and the root (it has $P_{\text {above }}$ as a strict subderivation) and whose derived judgment is identical to the one of $P_{\text {above }}$ except for the memory $M$ and the output set $S$.

Lemma 12 (Correct Memory). In a complete algorithmic derivation whose conclusion's memory is $M$, each subderivation of the form $M^{\prime} @ \Gamma ; \emptyset \vdash_{\mathrm{sat}}^{\mathrm{alg}} S: P_{\mathrm{at}}$ has a number of recurrent ancestors equal to $M^{\prime}\left(\Gamma \vdash P_{\mathrm{at}}\right)-M\left(\Gamma \vdash P_{\mathrm{at}}\right)$.

Proof. This is immediately proved by reasoning on the path from the start of the complete derivation to the subderivation. By construction of the algorithmic judgment, each judgment of the form $M^{\prime} @ \Gamma^{\prime} ; \emptyset \vdash_{\text {sat }}^{\text {alg }} S^{\prime}: Q_{\text {at }}$ is proved by either the rule ALG-SAT-KILL, which terminates the path with the invariant maintained, or the rule ALG-SAT-POST, which continues the path with the invariant preserved by incrementing the count in memory.

Lemma 13 (Recurrence Decrementation). If a saturated logic derivation contains $n+2$ occurences of the same judgment along a given path, then there is a valid saturated logic derivation with $n+1$ occurences of this judgment.

Proof. If $t$ is the proof term with $n+2$ occurences of the same judgment along a given path, let $u_{1}$ be the subterm corresponding to the very last occurence of the judgment, and $u_{2}$ the last-but-one. The term $t\left[u_{1} / u_{2}\right]$ is a valid proof term (of the same result as $t$ ), with only $n+1$ occurences of this same judgment.

Note that this transformation changes the computational meaning of the term - it must be used with care, as it could break unicity completeness. 
Theorem 5 (Provability completeness). If a memory $M$ contains multiplicities of either 0 or 1 (never 2 or more), then any algorithmic judgment with memory $M$ is complete for unicity: if the corresponding saturating judgment is inhabited, then the algorithmic judgment returns an inhabited 2-set.

Proof. If the saturating judgment $\Gamma ; \Gamma^{\prime} \vdash_{\text {sat }} t: A$ holds for a given $t$, we can assume without loss of generality that $t$ contains no two recurring occurences of the same judgment along any path - indeed, it suffices to repeatedly apply the Recurrence Decrementation Lemma 13 to obtain such a $t$ with no recurring judgment.

The proof of our result goes by induction on (the saturated derivation of) this no-recurrence $t$, mirroring each inference step into an algorithmic inference rule returning an inhabited set. Consider the following saturated rule for example:

$$
\frac{\Gamma \vdash u \Uparrow A}{\Gamma \vdash \sigma_{1} u \Uparrow A+B}
$$

We can build the corresponding algorithmic rule

$$
\frac{M^{\prime} @ \Gamma \vdash^{\mathrm{alg}} S_{1} \Uparrow A \quad M^{\prime} @ \Gamma \vdash^{\mathrm{alg}} S_{2} \Uparrow B}{M^{\prime} @ \Gamma \vdash^{\mathrm{alg}} \sigma_{1} S_{1} \cup_{2} \sigma_{2} S_{2} \Uparrow A+B}
$$

By induction hypothesis we have that $S_{1}$ is inhabited; from it we deduce that $\sigma_{1} \quad S_{1}$ is inhabited, and thus $\sigma_{1} S_{1} \cup_{2} \sigma_{2} \quad S_{2}$ is inhabited.

It would be tempting to claim that the resulting set is inhabited by $t$. That, in our example above, $u$ inhabits $S_{1}$ and thus $t=\sigma_{1} u$ inhabits $\sigma_{1} \quad S_{1} \cup_{2} \quad \sigma_{2} \quad S_{2}$. This stronger statement is incorrect, however, as the union of 2-sets may drop some inhabitants if it already has found two distinct terms.

The first difficulty in the induction are with judgments of the form $\Gamma ; \emptyset \vdash_{\text {sat }} u: P_{\text {at }}$ : to build an inhabited result set, we need to use the rule ALG-SAT-POST and thus check that $\Gamma \vdash P_{\text {at }}$ does not occur twice in the current memory $M^{\prime}$. By the Correct Memory Lemma 12 , we know that $M^{\prime}\left(\Gamma \vdash P_{\text {at }}\right)$ is the sum of the number of recurrent ancestors and of $M\left(\Gamma \vdash P_{\mathrm{at}}\right)$. By definition of $t$ (as a term with no repeated judgment), we know that $\Gamma \vdash P_{\text {at }}$ did not already occur in $t$ itself - the count of recurrent ancestors is 0 . By hypothesis on $M$ we know that $M\left(\Gamma \vdash P_{\text {at }}\right)$ is at most 1 , so the sum cannot be 2 or more.

The second and last subtlety happens at the SINV-END rule for $\Gamma ; \Gamma^{\prime} \vdash_{\text {sinv }} t: P_{\text {at }}$. We read saturated derivation of premise $\Gamma ; \Gamma^{\prime} \vdash_{\text {sat }} t: P_{\text {at }}$, but build an algorithmic derivation in the trimmed context $M @ \Gamma ;\left(\begin{array}{lll}\Gamma^{\prime} & -{ }_{2} & \Gamma\end{array}\right) \vdash_{\text {sat }}^{\text {alg }} S: P_{\text {at. }}$. It is not necessarily the case that $t$ is well-defined in this restricted context. But that is not an issue for inhabitation: the only variables removed from $\Gamma^{\prime}$ are those for which at least one variable of the same type appears in $\Gamma$. We can thus replace each use of a trimmed variable by another variable of the same time in $\Gamma$, and get a valid derivation of the exact same size.

Theorem 6 (Unicity completeness). If a memory $M$ contains multiplicities of 0 only, then any algorithmic judgment with memory $M$ is complete for unicity: if the corresponding saturating judgment has two distinct inhabitants, then the algorithmic judgment returns a 2-set of two distinct elements.

Proof. Consider a pair of distinct inhabitants $t \neq u$ of a given judgment. Without loss of generality, we can assume that $t$ has no judgment ocurring twice or more. (We cannot also assume that $u$ has no judgment occuring twice, as the no-recurrence reduction of a general $u$ may be equal to $t$.)

Without loss of generality, we will also assume that $t$ and $u$ use a consistent ordering for invertible rules (for example the one presented in the algorithmic judgment); this assumption can be made because reordering inference steps gives a term in the $\left(={ }_{\text {icc }}\right)$ equivalence class, that is thus $\beta \eta$-equivalent to the starting term.

Finally, to justify the SINV-END rule we need to invoke the "two or more" result of Section 4: without loss of generality we assume that $t$ and $u$ never use more than two variables of any given type (additional variables are weakened as soon as they are introduced). If $t$ and $u$ have distinct shapes (they are in disjoint equivalent classes of terms that erase to the same logic derivation), we immediately know that the disequality $t \neq u$ is preserved. If they have the same shape, we need to invoke the Counting approximation Corollary 1 to know that we can pick two distinct terms in this restricted space.

We then prove our result by parallel induction on $t$ and $u$ : the saturated judgment is inhabited by at least two distinct inhabitants. As long as their subterms start with the same syntactic construction, we keep inducing in parallel. Their head constructor may only differ in a non-invertible introduction or elimination rule (we assumed that invertible steps were performed in the same order), for example we may have

$$
\frac{\Gamma \vdash t^{\prime} \Uparrow A}{\Gamma \vdash \sigma_{1} t^{\prime} \Uparrow A+B} \quad \frac{\Gamma \vdash u^{\prime} \Uparrow B}{\Gamma \vdash \sigma_{2} u^{\prime} \Uparrow A+B}
$$

We then invoke the previous Provability Completeness Theorem 5 on $t^{\prime}$ and $u^{\prime}$ : we can build corresponding derivations $M^{\prime} @$ $\Gamma \vdash^{\text {alg }} S \Uparrow A$ and $M^{\prime} @ \Gamma \vdash^{\text {alg }} T \Uparrow B$ where $S$ and $T$ are inhabited, and thus $\sigma_{1} S \cup_{2} \sigma_{2} T$ is inhabited by at least two distinct terms. The memory hypothesis of the provability theorem is fulfilled: because we know that there are no repetitions in $t$, and that we iterated in parallel on the structures of $t$ and $u$, we know that each judgment was seen at most once during the parallel induction. As we assumed our starting memory was all 0 , the memory $M^{\prime}$ at the point where $t$ and $u$ differ is thus (by Lemma 12) of at most 1 for any judgment.

There is one difficulty during the parallel induction, which is the SINV-END case. We read a saturated derivations of premise $\Gamma ; \Gamma^{\prime} \vdash_{\text {sat }} t: P_{\text {at }}$ and $\Gamma ; \Gamma^{\prime} \vdash_{\text {sat }} u: P_{\text {at }}$, but build an algorithmic derivation in the trimmed context $M @ \Gamma ;\left(\Gamma^{\prime}-{ }_{2} \Gamma\right) \vdash_{\mathrm{sat}}^{\mathrm{alg}} S: P_{\mathrm{at}}$. This is why we restricted $t$ and $u$ to not use more than two different variables of each type, so that they remain well-typed under this restriction.

Corollary (Theorem 4). Our unicity-deciding algorithm is terminating and complete for unicity $\S 1.5$.

Proof. Our unicity-deciding algorithm takes a judgment $\Delta \vdash A$ and returns the 2-set $S$ uniquely determined by a complete algorithmic derivation of the judgment $\emptyset @ \emptyset ; \emptyset ; \Delta \vdash_{\text {inv }}^{\text {alg }} S: A-$ whose memory is empty. There always exists exactly one derivation (Lemma 9), and it is finite (Lemma 8). Our algorithm can compute the next rule to apply in finite time, and all derivations are finite, so the algorithm is terminating. This root judgment has an empty memory, hence it is complete for unicity (Theorem 6). 